\title{
A Multifamily GLRT for Oil Spill Detection
}

\author{
Antonio De Maio, Fellow, IEEE, Danilo Orlando, Senior Member, IEEE, \\ Luca Pallotta, Member, IEEE, and Carmine Clemente, Member, IEEE
}

\begin{abstract}
This paper deals with detection of oil spills from multipolarization synthetic aperture radar images. The problem is cast in terms of a composite hypothesis test aimed at discriminating between the polarimetric covariance matrix (PCM) equality (absence of oil spills in the tested region) and the situation where the region under test exhibits a PCM with at least an ordered eigenvalue smaller than that of a reference covariance. This last setup reflects the physical condition where the backscattering associated with the oil spills leads to a signal, in some eigendirections, weaker than the one gathered from a reference area where the absence of any oil slicks is a priori known. A multifamily generalized likelihood ratio test approach is pursued to come up with an adaptive detector ensuring the constant false alarm rate property. At the analysis stage, the behavior of the new architecture is investigated in comparison with a benchmark (but nonimplementable) structure and some other suboptimum adaptive detectors available in the open literature. This study, which is conducted in the presence of both simulated and real data, confirms the practical effectiveness of the new approach.
\end{abstract}

Index Terms-Constant false alarm rate (CFAR), covariance matrix equality, invariance, multifamily generalized likelihood ratio test (MGLRT), oil spill detection, one-sided generalized likelihood ratio test (GLRT).

\section{INTRODUCTION}

$\mathbf{O}$ IL spills represent a threat to the environment, wildlife, and to human life through the food chain. Natural phenomena, oil pipeline breakages, and illegal human activities are the causes of the presence of oil spills in the sea. In particular, illegal washing activities of oil tankers contribute $70 \%$ to sea pollution with oil [1]. As this kind of activity is difficult to prevent, monitoring and detection of the phenomenon is vital to be able to act timely and avoid natural disasters. Oil spills have peculiar characteristics that make them visible in synthetic aperture radar (SAR) images. Oil smooths the sea surface, reducing its roughness, thus appearing darker in SAR images.

Manuscript received March 14, 2016; revised May 30, 2016 and July 13, 2016; accepted July 15, 2016. Date of publication October 18, 2016; date of current version November 29, 2016. The work of C. Clemente was supported in part by the Engineering and Physical Sciences Research Council under Grant EP/K014307/1 and in part by the MOD University Defence Research Collaboration in Signal Processing.

A. De Maio is with the Dipartimento di Ingegneria Elettrica e delle Tecnologie dell'Informazione, Università degli Studi di Napoli Federico II, 80125 Napoli, Italy (e-mail: ademaio@unina.it).

D. Orlando is with the Faculty of Engineering, Università degli Studi "Niccolò Cusano," 00166 Roma, Italy (e-mail: danilo.orlando@unicusano.it).

L. Pallotta was with the National Laboratory for Multimedia Communications (CNIT), 43124 Parma, Italy. He is now with Università degli Studi di Napoli Federico II, 80125 Napoli, Italy (e-mail: luca.pallotta@unina.it).

C. Clemente is with the Centre for Excellence in Signal and Image Processing, Department of Electronic and Electrical Engineering, University of Strathclyde, Glasgow G1 1XW, U.K. (e-mail: carmine.clemente@ strath.ac.uk).

Color versions of one or more of the figures in this paper are available online at http://ieeexplore.ieee.org.

Digital Object Identifier 10.1109/TGRS.2016.2594867
For this reason, at least in principle, SAR represents a powerful tool for oil spill detection and sizing [2]. Improved reliability in such detection process has been demonstrated, exploiting the additional information residing in the polarimetric returns [3]. Recently, the topic of oil spill detection in polarimetric SAR has been investigated with different approaches. In [4], a feature extraction and maximum entropy segmentation approach has been proposed. The capabilities of hybrid/compact dual-polarization (dual-pol) modes have been assessed in [5], with experimental results suggesting that hybrid/compact and (HH, VV) dual-pol modes deliver better detection performance compared with conventional dual-pol modes, i.e., (HH, HV) and $(\mathrm{VH}, \mathrm{VV})$. The potential of circular polarization coherence and polarimetric anisotropy has been assessed in [6], showing that the anisotropy is always higher within the slick area both in L- and C-bands. Decision schemes ensuring the constant false alarm rate (CFAR) property and the target decomposition theorem have been jointly used in [7] to detect and classify oil spills, highlighting that entropy plays a key role in the classification process. Three techniques based on the generalized likelihood ratio test (GLRT) and the maximum-likelihood estimate have been proposed in [8], providing near-optimal performance in real environment. Compact polarimetric $(\mathrm{CP})$ mode has been proposed in [9] as an alternative transmission mode providing enhanced discriminating capabilities due to a specific X-Bragg scattering model. In [10], the CP mode has been also exploited; in particular, a quad-polarization representation of the data has been obtained from a circular transmit linear receive configuration and has been used for statistical detection of oil spills. Finally, future perspectives in oil slicks detection, including multisensor integration, have been suggested in [11].

In this paper, the problem of oil spill detection is formulated in terms of a binary hypothesis test aimed at discriminating between the presence and the absence of variations in the polarimetric covariance matrix (PCM) of the radar returns. The idea is to compare the region under test, which possibly contains oil spills, to a reference area where only echoes from the sea are present. As already stated, the presence of oil damps short gravity-capillary waves down and decreases the backscattering of sea surface. Thus, it is reasonable to assume that the PCM of data containing oil slicks shares eigenvalues smaller than or equal to the PCM of the sea returns. The decision problem is solved applying the GLRT, and the devised architecture is referred to as positive definite difference GLRT (PDD-GLRT). At the design stage, it is assumed that the rank difference between the two covariance matrices is known. However, this assumption might not be met in practical scenarios, since such a priori information is not available at the receiver. In order to circumvent this drawback, the previous 
results are extended to come up with a decision rule capable of properly estimating the rank difference. This goal is achieved exploiting the multifamily GLRT (MGLRT) [12], and the devised decision rule is referred to as the multifamily PDD-GLRT (M-PDD-GLRT). In addition, a discussion on the invariance properties of the PDD-GLRT (and, hence, of the M-PDDGLRT) is provided. More precisely, the invariance principle is invoked to express the decision statistic in terms of a maximal invariant [13], [14]. Finally, numerical examples are provided to prove the effectiveness of the proposed approach also in comparison with existing strategies for oil spill detection.

The remainder of this paper is organized as follows. Section II is devoted to the problem formulation. The derivations of the PDD-GLRT and its multifamily modification are reported in Section III, including details on the invariance and CFAR property. Section IV analyzes the performance in terms of detection probability on simulated and real data. Some concluding remarks and future research tracks are given in Section V. Finally, proofs and derivations are confined to the Appendixes.

Notation: Vectors and matrices are denoted by boldface lowercase and uppercase letters, respectively. Symbols $\operatorname{det}(\cdot)$ and $\operatorname{Tr}(\cdot)$ denote the determinant and the trace of a square matrix, respectively. Symbol $\mathbb{H}^{N}$ is used to represent the set of $N \times N$ Hermitian matrices, whereas $\boldsymbol{I}$ and $\mathbf{0}$ represent the identity matrix and the null vector or matrix, respectively, both of suitable dimensions. The curled inequality symbol $\succeq$ (and its strict form $\succ$ ) is used to denote generalized matrix inequality: for any $\boldsymbol{A} \in \mathbb{H}^{N}, \boldsymbol{A} \succeq \mathbf{0}$ means that $\boldsymbol{A}$ is a positive semidefinite matrix ( $\boldsymbol{A} \succ \mathbf{0}$ for positive definiteness). As to the numerical sets, $\mathbb{R}$ is the set of real numbers, $\mathbb{R}^{N \times M}$ is the set of $(N \times M)$-dimensional real matrices (or vectors if $M=1)$, $\mathbb{C}$ is the set of complex numbers, and $\mathbb{C}^{N \times M}$ is the set of $(N \times M)$ dimensional complex matrices (or vectors if $M=1$ ). Symbols $(\cdot)^{T}$ and $(\cdot)^{\dagger}$ stand for transpose and conjugate transpose, respectively. Finally, the acronym i.i.d. means independent and identically distributed, whereas symbol $E[\cdot]$ denotes statistical expectation.

\section{Problem Formulation}

A multipolarization SAR sensor, for each pixel of the image under test, collects $N_{0}=4$ complex returns from four different polarimetric channels (namely, $\mathrm{HH}, \mathrm{HV}, \mathrm{VH}$, and $\mathrm{VV}$ ). However, in the monostatic configuration, when both the scattering reciprocity and backscatter alignment convention hold, then the returns from channel HV coincide with those from channel VH [15]. For the aforementioned reason, in the sequel, returns from $N=3$ polarimetric channels are considered.

The returns associated with the same pixel are organized in the specific order $\mathrm{HH}, \mathrm{HV}$, and $\mathrm{VV}$ to form the vector $\boldsymbol{x}\left(l_{1}, l_{2}\right), l_{1}=1, \ldots, L_{1}$ and $l_{2}=1, \ldots, L_{2}\left(L_{1}\right.$ and $L_{2}$ represent the vertical and horizontal sizes of the image, respectively). Therefore, the sensor provides a 3-D data stack $\boldsymbol{X}$ of size $L_{1} \times L_{2} \times N$, which is referred to as a data cube in the following, whose pictorial representation is given in Fig. 1 .

Starting from the data cube $\boldsymbol{X}$ of the scene illuminated by the radar, for each pixel under test, we extract a rectangular

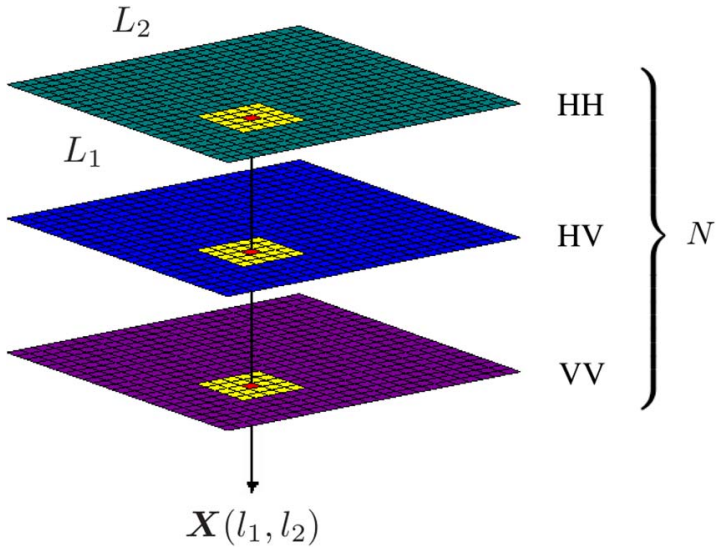

Fig. 1. Pictorial representation of the construction of the data cube for polarimetric images.

neighborhood $\mathcal{A}$ of size $K=W_{1} \times W_{2} \geq N$. We denote by $\boldsymbol{Z}=\left[\begin{array}{lll}\boldsymbol{z}_{1} & \ldots & \boldsymbol{z}_{K}\end{array}\right] \in \mathbb{C}^{N \times K}$ the matrix whose columns are the vectors of the polarimetric returns from the pixels of $\boldsymbol{X}$ which fall in the region $\mathcal{A}$. The matrix $\boldsymbol{Z}$ is modeled as a random matrix, whose columns are assumed i.i.d. random vectors drawn from a complex circular zero-mean Gaussian distribution with positive definite covariance matrix $\boldsymbol{R}$.

Our goal is to identify those regions of $\boldsymbol{X}$ that exhibit variations of the covariance matrix $\boldsymbol{R}$ with respect to a preassigned reference region. Specifically, the presence of specific objects in the observed scene yields signal echoes with spectral properties different from those associated with the background. For instance, consider an oil spill on the sea surface, whose reflectivity coefficient reduces the intensity of the backscattering signal. Thus, if we denote by $\boldsymbol{R}_{\mathcal{A}_{1}}$ and $\boldsymbol{R}_{\mathcal{A}_{2}}$ the PCMs of a region $\mathcal{A}_{1}$ containing sea returns and of a region $\mathcal{A}_{2}$ associated with an oil spill, respectively, physical argumentations on backscattering lead to the condition $\boldsymbol{R}_{\mathcal{A}_{1}}-\boldsymbol{R}_{\mathcal{A}_{2}} \succeq \mathbf{0}$.

Based on the aforementioned observations, the detection problem addressed in this paper can be written in terms of the following hypothesis test:

$$
\left\{\begin{array}{l}
H_{0}: \begin{cases}\boldsymbol{z}_{k} \sim C \mathcal{N}(\mathbf{0}, \boldsymbol{R}), & k=1, \ldots, K \\
\boldsymbol{y}_{m} \sim C \mathcal{N}(\mathbf{0}, \boldsymbol{R}), & m=1, \ldots, M\end{cases} \\
H_{1}: \begin{cases}\boldsymbol{z}_{k} \sim C \mathcal{N}\left(\mathbf{0}, \boldsymbol{R}_{1}\right), & k=1, \ldots, K \\
\boldsymbol{y}_{m} \sim C \mathcal{N}\left(\mathbf{0}, \boldsymbol{R}_{1}+\boldsymbol{R}_{2}\right), & m=1, \ldots, M\end{cases}
\end{array}\right.
$$

where $\boldsymbol{z}_{k}, k=1, \ldots, K$, and $\boldsymbol{y}_{m}, m=1, \ldots, M$, are statistically independent random vectors ${ }^{1}$; specifically, $\boldsymbol{y}_{m}$ contains the echoes from the reference area (namely, the clean sea in the context of oil spill detection) which has a size of $M$ pixels, whereas $\boldsymbol{z}_{k}$ are the $K$ pixels under test (possibly containing oil spills). Matrices $\boldsymbol{R}$ and $\boldsymbol{R}_{1}$ are full rank, i.e., $\operatorname{Rank}(\boldsymbol{R})=$ $\operatorname{Rank}\left(\boldsymbol{R}_{1}\right)=N$. The data covariance matrix for the case of active sensing cannot be singular due to the presence of filtered thermal noise (due to electronic devices composing the system

${ }^{1}$ For the sake of simplicity, in the following, we neglect the possible low correlation level between adjacent pixels. 


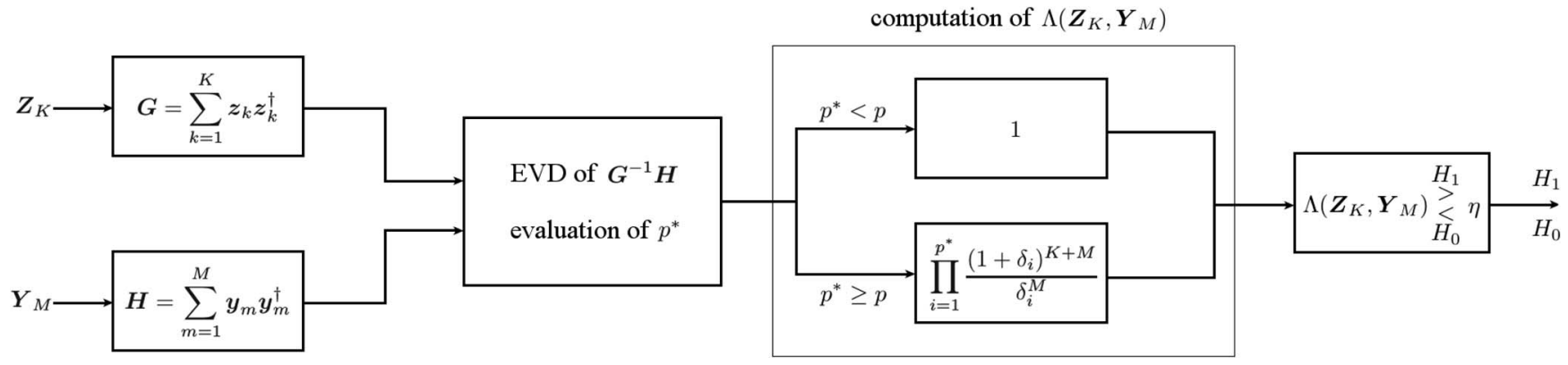

Fig. 2. Block scheme of the PDD-GLRT detector.

chain) whose power puts a positive lower bound to the covariance matrix eigenvalues. Moreover, as shown in [16, Ch. 4], the circular Gaussian assumption (and, hence, full-rank PCM) has been experimentally shown to be valid for polarimetric SAR data. The rank of $\boldsymbol{R}_{2}, p$, for example, is assumed to be known and within the interval $(0, N]$.

It is clear that, in the context of oil spill detection, the null hypothesis $H_{0}$ represents the clean sea situation, whereas the alternative one refers to the presence of oil spills. Finally, we assume that $K \geq N$ and $M \geq N$ to ensure that the sample PCMs

$$
\boldsymbol{G}=\sum_{k=1}^{K} \boldsymbol{z}_{k} \boldsymbol{z}_{k}^{\dagger} \quad \boldsymbol{H}=\sum_{m=1}^{M} \boldsymbol{y}_{m} \boldsymbol{y}_{m}^{\dagger}
$$

are full rank with probability 1 . As already stated, the estimated covariance matrices (i.e., sample covariance matrices) are nonsingular with probability 1 [17] provided that $K \geq 3$ for three polarimetric channels (or $K \geq 2$ for two polarizations). Moreover, if the sample covariance matrix would be close to singularity, a diagonal loading procedure could be applied to regularize it. The proposed procedure can be thus applied after regularization.

\section{Detector Design}

Here, an adaptive decision rule is devised resorting to the GLRT design criterion. To this end, let us define $\boldsymbol{Z}_{K}=$ $\left[\begin{array}{lll}\boldsymbol{z}_{1} & \ldots & \boldsymbol{z}_{K}\end{array}\right]$ and $\boldsymbol{Y}_{M}=\left[\begin{array}{lll}\boldsymbol{y}_{1} & \ldots & \boldsymbol{y}_{M}\end{array}\right]$, and then the likelihood functions of $\boldsymbol{Z}_{K}$ and $\boldsymbol{Y}_{M}$ under $H_{0}$ and $H_{1}$ are given by

$$
\begin{aligned}
& f\left(\boldsymbol{Z}_{K}, \boldsymbol{Y}_{M} ; \boldsymbol{R}, H_{0}\right) \\
& =\left[\frac{1}{\pi^{N} \operatorname{det}(\boldsymbol{R})}\right]^{K+M} \exp \left\{-\operatorname{tr}\left[\boldsymbol{R}^{-1}(\boldsymbol{G}+\boldsymbol{H})\right]\right\} \\
& f\left(\boldsymbol{Z}_{K}, \boldsymbol{Y}_{M} ; \boldsymbol{R}_{1}, \boldsymbol{R}_{2}, H_{1}\right) \\
& =\left[\frac{1}{\pi^{N}}\right]^{K+M} \frac{\exp \left\{-\operatorname{tr}\left[\boldsymbol{R}_{1}^{-1} \boldsymbol{G}\right]-\operatorname{tr}\left[\left(\boldsymbol{R}_{1}+\boldsymbol{R}_{2}\right)^{-1} \boldsymbol{H}\right]\right\}}{\left[\operatorname{det}\left(\boldsymbol{R}_{1}\right)\right]^{K}\left[\operatorname{det}\left(\boldsymbol{R}_{1}+\boldsymbol{R}_{2}\right)\right]^{M}}
\end{aligned}
$$

respectively. Now, observe that the GLRT for the problem at hand is

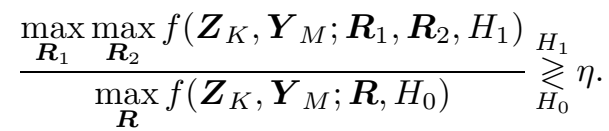

The following proposition provides closed-form expression for a test statistically equivalent to (5).

Proposition 3.1: The generalized likelihood ratio (5) is statistically equivalent to

$$
\Lambda_{p}\left(\boldsymbol{Z}_{K}, \boldsymbol{Y}_{M}\right) \underset{H_{0}}{\stackrel{H_{1}}{\gtrless}} \eta
$$

where

$$
\Lambda_{p}\left(\boldsymbol{Z}_{K}, \boldsymbol{Y}_{M}\right)= \begin{cases}1, & \text { if } p^{*}<p \\ \prod_{i=1}^{p^{*}} \frac{\left(1+\delta_{i}\right)^{K+M}}{\delta_{i}^{M}}, & \text { otherwise }\end{cases}
$$

$\delta_{i}, i=1, \ldots, p^{*}$, are $p^{*}$ eigenvalues of $\boldsymbol{G}^{-1} \boldsymbol{H}$, with $p^{*}$ being the minimum between the number of eigenvalues of $\boldsymbol{G}^{-1} \boldsymbol{H}$ greater than $M / K$ and $p$.

Proof: See Appendix I.

The preceding architecture will be referred to in the following as the PDD-GLRT. In Fig. 2, a block scheme of the PDD-GLRT is depicted. Specifically, the first step consists in the computation of the sample covariance matrices $\boldsymbol{G}$ and $\boldsymbol{H}$ using data $\boldsymbol{Z}_{K}$ and $\boldsymbol{Y}_{M}$, respectively. Then, the eigenvalue decomposition (EVD) of the product $\boldsymbol{G}^{-1} \boldsymbol{H}$ is evaluated, as well as $p^{*}$. Finally, the statistic $\Lambda_{p}\left(\boldsymbol{Z}_{K}, \boldsymbol{Y}_{M}\right)$ is constructed and compared with a threshold to perform the decision.

In the next subsection, we apply the exponential embedded family (EEF) framework [12], [18] to devise an architecture based upon the PDD-GLRT that does not use any a priori information on the rank of $\boldsymbol{R}_{2}$.

\section{A. M-PDD-GLRT}

In practical situations, the exact knowledge about the rank of $\boldsymbol{R}_{2}$ could not be available, and hence, it has to be somehow estimated. To this end, here, we introduce a variant of the PDD-GLRT which does not need the aforementioned a priori information. Specifically, the binary hypotheses considered before become multiple nested instances, each tied up to a rank value of $\boldsymbol{R}_{2}$, which is denoted by $r(i)=i, i=1, \ldots, N$. In this scenario, the classical GLRT cannot be used, and hence, we resort to the MGLRT [12], [18] that allows the PDD-GLRT to be employed also in the case where the rank is not known. 


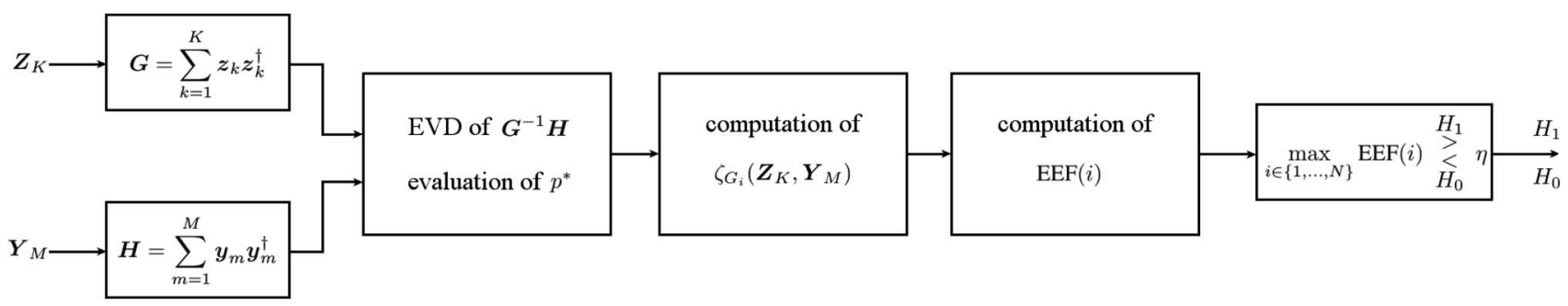

Fig. 3. Block scheme of the M-PDD-GLRT detector.

Specifically, the MGLRT can be written in terms of the EEF computed for a given model $\operatorname{order}^{2} i, i=1, \ldots, N$, [18], i.e.,

$$
\max _{i \in\{1, \ldots, N\}} \operatorname{EEF}(i) \underset{H_{0}}{\stackrel{H_{1}}{\gtrless}} \eta
$$

where

$$
\begin{aligned}
\operatorname{EEF}(i)=\left(\zeta_{G_{i}}\left(\boldsymbol{Z}_{K}, \boldsymbol{Y}_{M}\right)-r(i)\left[\log \left(\frac{\zeta_{G_{i}}\left(\boldsymbol{Z}_{K}, \boldsymbol{Y}_{M}\right)}{r(i)}\right)+1\right]\right) \\
\times u\left(\frac{\zeta_{G_{i}}\left(\boldsymbol{Z}_{K}, \boldsymbol{Y}_{M}\right)}{r(i)}-1\right), \quad i=1, \ldots, N
\end{aligned}
$$

In the last equation, $\zeta_{G_{i}}\left(\boldsymbol{Z}_{K}, \boldsymbol{Y}_{M}\right)$ is two times the natural logarithm of the ratio between the compressed likelihood functions, i.e.,

$$
\zeta_{G_{i}}\left(\boldsymbol{Z}_{K}, \boldsymbol{Y}_{M}\right)=2 \log \frac{f\left(\boldsymbol{Z}_{K}, \boldsymbol{Y}_{M} ; \hat{\boldsymbol{R}}_{1}, \hat{\boldsymbol{R}}_{2}, H_{1}\right)}{f\left(\boldsymbol{Z}_{K}, \boldsymbol{Y}_{M} ; \hat{\boldsymbol{R}}, H_{0}\right)}
$$

where, under the assumption that $p=i$, the numerator of the logarithm argument is given by (54), whereas the denominator is defined by (55); finally, $u(\cdot)$ is the Heaviside step function. Thus, when there are multiple nested alternative hypotheses, the MGLRT first computes the PDD-GLRT for each hypothesis; $\zeta_{G_{i}}(\cdot)$, for example, applies the transformation (9) to construct the $\operatorname{EEF}(\mathrm{i})$ and chooses the hypothesis attaining the maximum. For the sake of completeness, we provide in the following the explicit expression of $\zeta_{G_{i}}\left(Z_{K}, \boldsymbol{Y}_{M}\right)$, which contains the constants which have been incorporated into the threshold of the PDD-GLRT, i.e., $\zeta_{G_{i}}\left(\boldsymbol{Z}_{K}, \boldsymbol{Y}_{M}\right)=0$ if $p^{*}<p$ and

$$
\begin{aligned}
\zeta_{G_{i}}\left(\boldsymbol{Z}_{K}, \boldsymbol{Y}_{M}\right)= & -2(K+M) r(i) \log (K+M) \\
& +2 K r(i) \log (K)+2 M r(i) \log (M) \\
& -2 M \sum_{n=1}^{r(i)} \log \delta_{i}+2(K+M) \sum_{n=1}^{r(i)} \log \left(1+\delta_{i}\right)
\end{aligned}
$$

otherwise.

Decision scheme (8) will be referred to in the following as the M-PDD-GLRT. A pictorial description of this receiver is given in Fig. 3. As shown for the PDD-GLRT, the first steps consist in computing the sample covariance matrices $\boldsymbol{G}$ and $\boldsymbol{H}$ from data and in performing the EVD of $\boldsymbol{G}^{-1} \boldsymbol{H}$.

\footnotetext{
${ }^{2}$ The model order is represented by the rank of $\boldsymbol{R}_{2}$.
}

Then, $\forall i=1, \ldots, N, p^{*}$ is used to construct $\zeta_{G_{i}}\left(\boldsymbol{Z}_{K}, \boldsymbol{Y}_{M}\right)$. Finally, the M-PDD-GLRT performs the decision, choosing the maximum of $\zeta_{G_{i}}\left(\boldsymbol{Z}_{K}, \boldsymbol{Y}_{M}\right), i=1, \ldots, N$, and comparing it to a threshold.

\section{B. Invariance}

Before concluding this section, some interesting properties of the obtained decision statistics relying on the invariance principle [13], [14] are given. More precisely, consider the group of transformations $(\mathcal{D}, \cdot)$, where $\mathcal{D}$ is the set of nonsingular $N$-dimensional matrices, and $\cdot$ is the matrix product, acting on $\boldsymbol{G}$ and $\boldsymbol{H}$ (which represent a sufficient statistic) as follows:

$$
\boldsymbol{G} \rightarrow \boldsymbol{D G} \boldsymbol{D}^{\dagger}, \quad \boldsymbol{H} \rightarrow \boldsymbol{D H} \boldsymbol{D}^{\dagger}, \quad \boldsymbol{D} \in \mathcal{D} .
$$

Then, a maximal invariant statistic for problem (1) and with respect to the aforementioned group of transformations is the $N$-dimensional vector whose entries are the eigenvalues of

$$
\boldsymbol{G}^{-1} H
$$

which are denoted by $\delta_{1}, \ldots, \delta_{N}$ [19]. It follows that both the PDD-GLRT and the M-PDD-GLRT are invariant to the aforementioned group of transformations. This invariance allows claiming that both detectors possess the CFAR property with respect to the covariance matrix $\boldsymbol{R}$ under the $H_{0}$ hypothesis.

Remarkably, data transformation induces parameter transformation, and as a consequence, it is possible to derive a maximal invariant statistic in the parameter space, which reduces the dimensionality of the latter. Following the lead of [19], an induced maximal invariant statistic would be an $N$-dimensional vector whose entries are the eigenvalues of

$$
\boldsymbol{R}_{1}^{-1}\left(\boldsymbol{R}_{1}+\boldsymbol{R}_{2}\right)
$$

However, given the rank of $\boldsymbol{R}_{2}, N-p$ eigenvalues of the matrix (14) are equal to 1 and can be neglected. As a consequence, in the current case, an induced maximal invariant statistic is a $p$-dimensional vector composed of the eigenvalues of (14) which are greater than 1 , i.e., $\lambda_{1}, \ldots, \lambda_{p}$.

It is clear that the induced maximal invariant reduces the dimensionality of the parameter space and measures in what extent the difference between the covariance matrices is positive definite. Thus, it can be used to evaluate the performances of the proposed algorithm. To gain additional insights, it is worth deriving its explicit expressions for the cases $p=1$ and $p=2$. 
In particular, when $p=1$, it can be shown that an induced maximal invariant is given by

$$
|\alpha|^{2} \boldsymbol{p}^{\dagger} \boldsymbol{R}_{1}^{-1} \boldsymbol{p}
$$

where $\alpha \in \mathbb{C}, \alpha \neq 0$, and $\boldsymbol{p} \in \mathbb{C}^{N \times 1}, \boldsymbol{p} \neq \mathbf{0}$. Equation (15) can be interpreted as a measure of the level of positive definiteness of $\boldsymbol{R}_{1}+\boldsymbol{R}_{2}$ or, generally speaking, as a measure of the signalto-noise ratio (SNR). In the case $p=2$, an induced maximal invariant is a 2-D statistic composed of the eigenvalues of

$$
\boldsymbol{P}^{\dagger} \boldsymbol{R}_{1}^{-1} \boldsymbol{P}
$$

where $\boldsymbol{P}=\left[\alpha \boldsymbol{p}_{1} \beta \boldsymbol{p}_{2}\right]$, with $\alpha, \beta \in \mathbb{C}, \alpha \neq 0 \neq \beta$, and $\boldsymbol{p}_{1} \in$ $\mathbb{C}^{N \times 1}$ linearly independent of $\boldsymbol{p}_{2} \in \mathbb{C}^{N \times 1}, \quad \boldsymbol{p}_{1} \neq \mathbf{0} \neq \boldsymbol{p}_{2}$. Again, such eigenvalues measure the level of positive definiteness and, hence, the SNR along two directions. The proofs of the preceding statements are provided in Appendix II.

\section{Results}

Here, we investigate the performances of the proposed detectors in terms of the probability of detection $P_{D}$. For comparison purposes, we also plot the performance curves of other approaches available in the open literature. More precisely, we consider the GLRT devised in [8], [20], and [21], whose expression is

$$
\Lambda_{\mathrm{GLRT}}=\frac{[\operatorname{det}(\boldsymbol{G}+\boldsymbol{H})]^{(K+M)}}{[\operatorname{det}(\boldsymbol{G})]^{K}[\operatorname{det}(\boldsymbol{H})]^{M}} \underset{H_{0}}{\gtrless} \eta
$$

the maximum-likelihood detector (MLD) proposed in [8], which is given by

$$
\Lambda_{\mathrm{MLD}}=\frac{\operatorname{det}(\boldsymbol{H})}{\operatorname{det}(\boldsymbol{G})} \underset{H_{0}}{\stackrel{H_{1}}{\gtrless}} \eta
$$

and the single likelihood detector (SLD) proposed in [8], whose decision statistic is

$$
\Lambda_{\mathrm{SLD}}=\operatorname{tr}\left(\boldsymbol{G}^{-1} \boldsymbol{H}\right) .
$$

In order to better analyze the behavior of all these detectors, the comparisons also include the clairvoyant counterparts, namely, the likelihood ratio test (LRT) and the clairvoyant SLD (C-SLD).

More precisely, the former is statistically equivalent to

$\Lambda_{\mathrm{LRT}}=\operatorname{tr}\left[\boldsymbol{R}^{-1}(\boldsymbol{G}+\boldsymbol{H})-\boldsymbol{R}_{1}^{-1} \boldsymbol{G}-\left(\boldsymbol{R}_{1}+\boldsymbol{R}_{2}\right)^{-1} \boldsymbol{H}\right] \underset{H_{0}}{\stackrel{H_{1}}{\gtrless}} \eta$

whereas the latter has the following expression:

$$
\Lambda_{\mathrm{C}-\mathrm{SLD}}=\operatorname{tr}\left(\boldsymbol{R}_{1}^{-1} \boldsymbol{H}\right) .
$$

The analysis is conducted on both simulated and real SAR data. In the latter case, we prove the effectiveness of the newly proposed detectors in terms of the capability to detect oil spills over the sea surface.

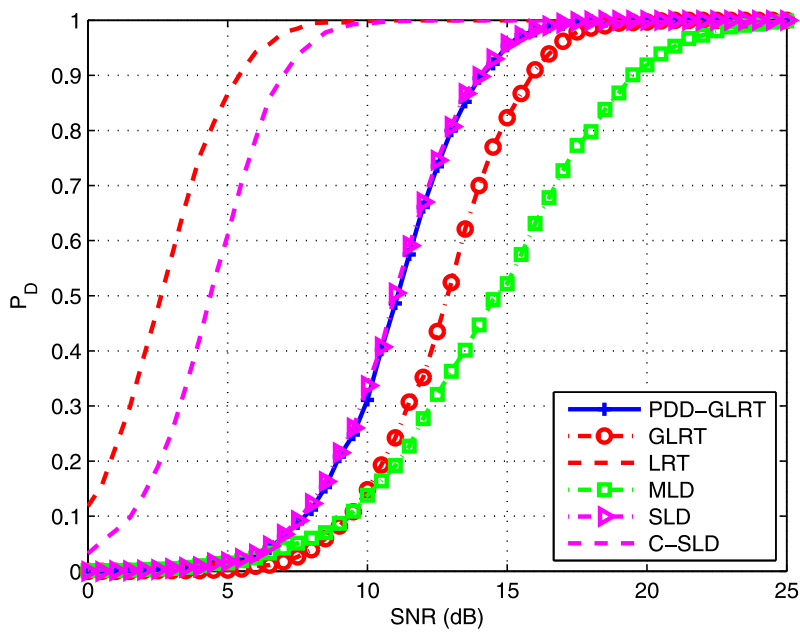

Fig. 4. $P_{D}$ versus SNR for (blue solid curve marked with + ) PDD-GLRT, (red dot-dashed curve marked with o) GLRT, (red dashed curve) LRT, (green dot-dashed curve marked with $\square$ ) MLD, (magenta dot-dashed curve marked with $\triangleright$ ) SLD, and (magenta dashed curve) C-SLD, for a nominal $P_{\mathrm{FA}}=10^{-4}$. The other simulation parameters are $K=9, M=9, N=3$, and $p=1$.

\section{A. Detection Capability on Simulated Data}

This subsection is devoted to the performance analysis using computer-simulated data for a preassigned value of probability of false alarm $P_{\mathrm{FA}}$. Specifically, the numerical examples are obtained by means of standard Monte Carlo counting techniques. The detection thresholds and the $P_{D}$ values are evaluated resorting to $100 / P_{\mathrm{FA}}$ and $10^{3}$ independent trials, respectively. The nominal $P_{\mathrm{FA}}$ is set to $10^{-4}$. As to the vector size, it is chosen equal to 3 , as stated at the beginning of Section II, whereas two values of parameter $p$ are considered, namely, $p=1$ and $p=2$. Recall that the performance parameter is represented by the induced maximal invariant which has been previously evaluated for the aforementioned cases and can be interpreted as the SNR.

The simulated data $\boldsymbol{z}_{k}, k=1, \ldots, K$, and $\boldsymbol{y}_{m}, m=$ $1, \ldots, M$, are modeled as $N$-dimensional zero-mean complex circular Gaussian vectors with covariance matrices $\boldsymbol{R}=\boldsymbol{R}_{1}=$ $\boldsymbol{I}$ and $\boldsymbol{R}_{1}+\boldsymbol{R}_{2}$, respectively. Matrix $\boldsymbol{R}_{2}$ is rank deficient and is defined as in (58) and (61) for the cases $p=1$ and $p=2$, respectively, where the $N$-dimensional steering vectors $\boldsymbol{p}, \boldsymbol{p}_{1}$, and $\boldsymbol{p}_{2}$ have been chosen as

$$
\begin{aligned}
\boldsymbol{p} & =[1,0, \ldots, 0]^{T} \\
\boldsymbol{p}_{1} & =[1,0, \ldots, 0]^{T} \\
\boldsymbol{p}_{2} & =[0,1,0, \ldots, 0]^{T} .
\end{aligned}
$$

Finally, $|\alpha|^{2}=|\beta|^{2}$.

Let us start from the case $p=1$ and recall that the SNR is given by (15). In Fig. 4, we plot the $P_{D}$ versus SNR, assuming $K=M=9$. Notice that the PDD-GLRT and the SLD achieve the best performance, overcoming all the other nonclairvoyant receivers. In fact, the PDD-GLRT reaches the value $P_{D}=0.9$ for an SNR approximately equal to $13 \mathrm{~dB}$, whereas the GLRT achieves the same $P_{D}$ value when the SNR is about equal to $16 \mathrm{~dB}$. The MLD attains the worst performance; in fact, it 


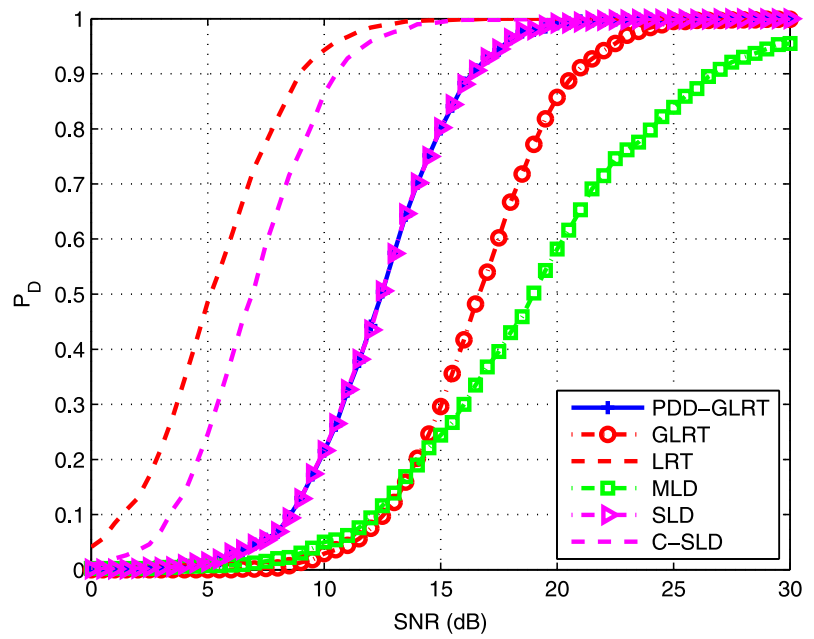

Fig. 5. $P_{D}$ versus SNR for (blue solid curve marked with +) PDD-GLRT, (red dot-dashed curve marked with o) GLRT, (red dashed curve) LRT, (green dot-dashed curve marked with $\square$ ) MLD, (magenta dot-dashed curve marked with $\triangleright$ ) SLD, and (magenta dashed curve) C-SLD, for a nominal $P_{\mathrm{FA}}=10^{-4}$. The other simulation parameters are $K=9, M=4, N=3$, and $p=1$.

achieves the $P_{D}$ value of 0.9 at SNR greater than $19 \mathrm{~dB}$. This figure does not contain the curve related to the M-PDD-GLRT because it shares exactly the same performance as the PDDGLRT since the rank is set to 1 . As expected, the clairvoyant decision schemes are superior to the respective adaptive counterparts. Specifically, at $P_{D}=0.9$ the PDD-GLRT suffers a loss of about 8.5 and $7 \mathrm{~dB}$ with respect to the LRT and the C-SLD, respectively.

In Fig. 5, we suppose that the number of test data $\boldsymbol{y}_{m}$, $m=1, \ldots, M$, is decreased, i.e., $M=4$, leaving $K=9$. Inspection of the figure highlights a performance degradation of all the receivers, due to the smaller amount of test data used for covariance estimation. The hierarchy observed in Fig. 4 is confirmed here. Again, the PDD-GLRT and the SLD share similar performances; their losses with respect to the LRT and to the C-SLD are kept within 7.5 and $6 \mathrm{~dB}$, respectively.

Finally, Fig. 6 assumes $K=4$ and $M=9$, i.e., we reduce the number of the training data. In this figure, we can observe the same trend as in Fig. 4, except for a significant performance degradation of all the considered architectures. Again, note that also for this parameter setting, the PDD-GLRT and the SLD show similar performances. The comparisons in Figs. 4-6 highlight that the performance degradation due to the scarce number of training samples is more significant than that introduced by a limited number of test samples.

Summarizing, the advantage of the PDD-GLRT with respect to the GLRT and the MLD seems clear. Moreover, when the number of test data $M$ is low (due, for instance, to the presence of strong heterogeneity in the data), we observe a severe loss in the MLD performance. Finally, we have also seen that, in the case of a rank-1 scenario, the PDD-GLRT has a behavior quite similar to that of the SLD with a slight gain over it.

The second part of the present analysis focuses on the case $p=2$, i.e., when the covariance $\boldsymbol{R}_{2}$ is a rank-2 matrix. The SNR is defined as

$$
\mathrm{SNR}=|\alpha|^{2} p_{1}^{\dagger} \boldsymbol{R}_{1}^{-1} \boldsymbol{p}_{1}+|\beta|^{2} \boldsymbol{p}_{2}^{\dagger} \boldsymbol{R}_{1}^{-1} \boldsymbol{p}_{2}=2|\alpha|^{2} .
$$

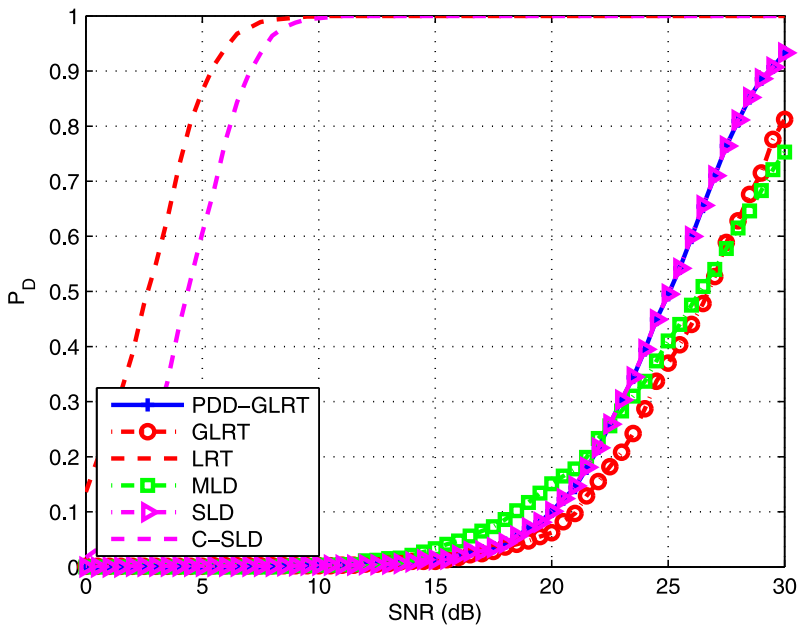

Fig. 6. $P_{D}$ versus SNR for (blue solid curve marked with + ) PDD-GLRT, (red dot-dashed curve marked with o) GLRT, (red dashed curve) LRT, (green dot-dashed curve marked with $\square$ ) MLD, (magenta dot-dashed curve marked with $\triangleright$ ) SLD, and (magenta dashed curve) C-SLD, for a nominal $P_{\mathrm{FA}}=10^{-4}$. The other simulation parameters are $K=4, M=9, N=3$, and $p=1$.

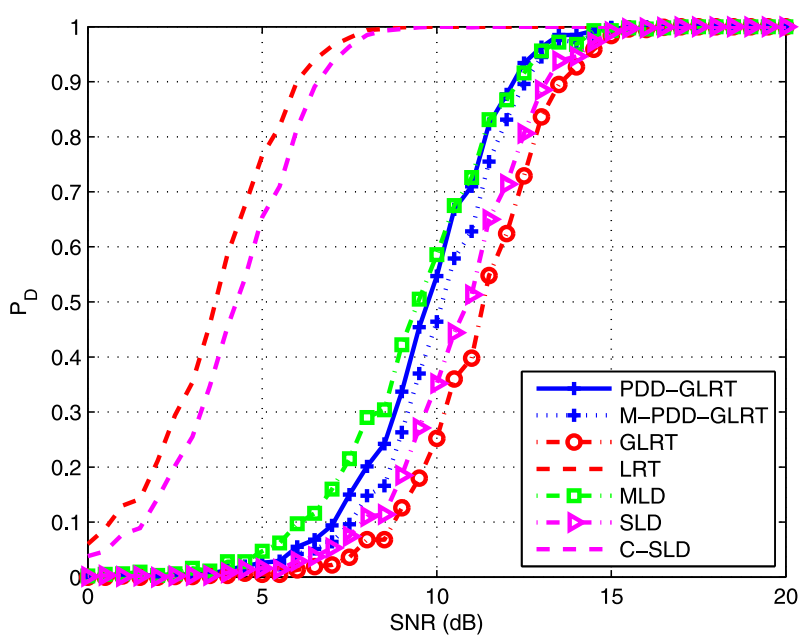

Fig. 7. $P_{D}$ versus SNR for (blue solid curve marked with + ) PDD-GLRT, (blue dotted curve marked with + ) M-PDD-GLRT, (red dot-dashed curve marked with $\circ$ ) GLRT, (red dashed curve) LRT, (green dot-dashed curve marked with $\square$ ) MLD, (magenta dot-dashed curve marked with $\triangleright$ ) SLD, and (magenta dashed curve) C-SLD, for a nominal $P_{\mathrm{FA}}=10^{-4}$. The other simulation parameters are $K=9, M=9, N=3$, and $p=2$.

In Fig. 7, we plot the $P_{D}$ versus SNR assuming $K=M=9$. It turns out that the PDD-GLRT and the MLD perform better than all the other adaptive architectures. In addition, the M-PDDGLRT exhibits some losses with respect to the PDD-GLRT, although both detectors outperform the GLRT and the SLD with a gain of about $1 \mathrm{~dB}$ at $P_{D}=0.9$. As a final remark, note that the PDD-GLRT suffers a detection loss of about $6.5 \mathrm{~dB}$ with respect to the LRT (and $5.5 \mathrm{~dB}$ with respect to the C-SLD).

In Fig. 8, we focus on a smaller number of test data, i.e., $M=4$, leaving the same value of $K$ as in Fig. 7. As observed for $p=1$, as well as for $p=2$, there is a general worsening in the receivers' performance. The figure highlights also that the PDD-GLRT and the M-PDD-GLRT achieve the best performances, with the former performing better than the latter. 


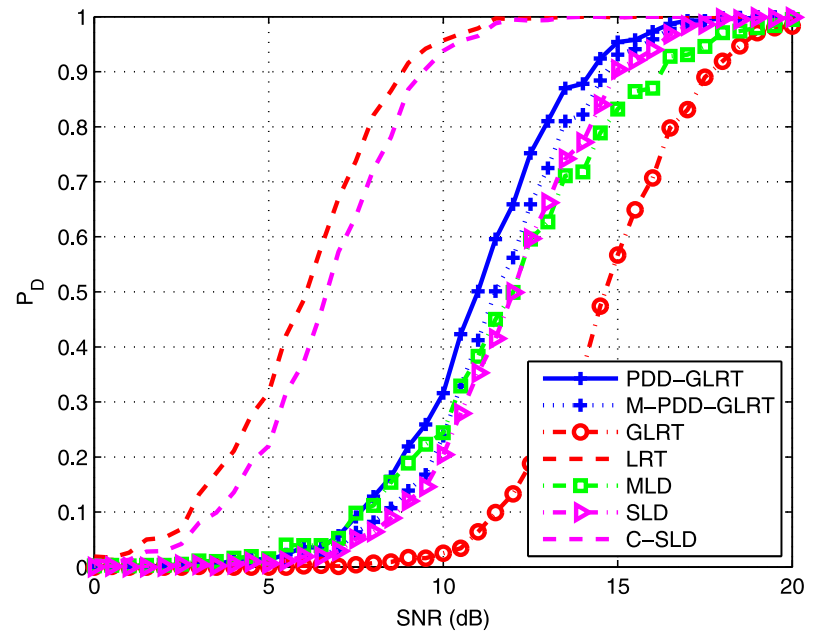

Fig. 8. $P_{D}$ versus SNR for (blue solid curve marked with + ) PDD-GLRT, (blue dotted curve marked with + ) M-PDD-GLRT, (red dot-dashed curve marked with o) GLRT, (red dashed curve) LRT, (green dot-dashed curve marked with $\square$ ) MLD, (magenta dot-dashed curve) SLD, and (magenta dashed curve marked with $\triangleright$ ) C-SLD, for a nominal $P_{\mathrm{FA}}=10^{-4}$. The other simulation parameters are $K=9, M=4, N=3$, and $p=2$.

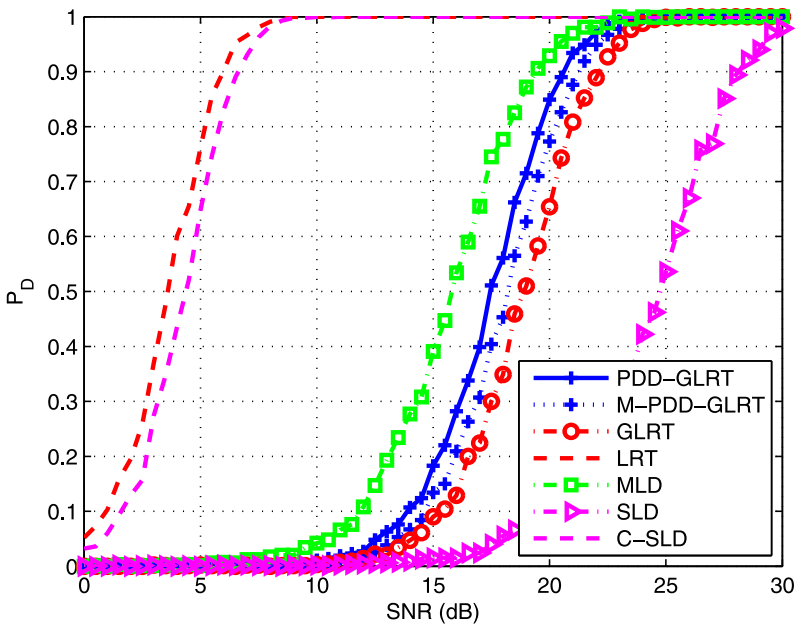

Fig. 9. $P_{D}$ versus SNR for (blue solid curve marked with +) PDD-GLRT, (blue dotted curve marked with + ) M-PDD-GLRT, (red dot-dashed curve marked with o) GLRT, (red dashed curve) LRT, (green dot-dashed curve marked with $\square$ ) MLD, (magenta dot-dashed curve marked with $\triangleright$ ) SLD, and (magenta dashed curve) C-SLD, for a nominal $P_{\mathrm{FA}}=10^{-4}$. The other simulation parameters are $K=4, M=9, N=3$, and $p=2$.

Finally, the GLRT is the worst, with a loss higher than $3 \mathrm{~dB}$, if compared with the PDD-GLRT and its multifamily version.

To conclude this analysis, in Fig. 9, we plot the performance curves for $K=4$ and $M=9$. Under this simulation setup, the best detector is the MLD, followed by the PDD-GLRT, the MPDD-GLRT, and the GLRT, whereas the SLD exhibits very poor performance.

Summarizing, the PDD-GLRT seems a reasonable choice since it exhibits a more robust behavior than the counterparts, being the one less sensitive to parameter variations and providing in all the analyzed situations acceptable performances. In other words, the PDD-GLRT represents a kind of an "all-season" architecture.

\section{B. Sensitivity Analysis to Variations of the Two-Dimensional Induced Maximal Invariant}

Here, we evaluate the sensitivity of the considered adaptive detectors to the eigenvalues of the matrix $\boldsymbol{R}_{1}^{-1} \boldsymbol{R}_{2}$, when $p=2$. In other words, we investigate the detection performance varying the aforementioned eigenvalues from very low to very high values. When one of them is very low, it is reasonable to consider the matrix $\boldsymbol{R}_{2}$ as a rank-1 matrix instead of a rank- 2 one. In other words, this simulation setup encompasses the case $p=1$ or $p=2$ according to the pair of eigenvalues. Thus, we consider here only the M-PDD-GLRT since the PDDGLRT assumes the knowledge of the rank of $\boldsymbol{R}_{2}$.

All the illustrative examples contain curves of $P_{D}$ versus the aforementioned eigenvalues, whereas the thresholds are set to ensure $P_{\mathrm{FA}}=10^{-4}$. Data vectors are generated as zero-mean complex circular Gaussian vectors with the covariance $\boldsymbol{R}_{1}=\boldsymbol{I}$, whereas the covariance $\boldsymbol{R}_{2}$ has been defined as a rank-2 matrix with a pair of eigenvalues given by $l_{1}$ and $l_{2}$. Since the induced maximal invariant is a bidimensional vector, ${ }^{3}\left[l_{1}, l_{2}\right]^{T}$, it is clear that a complete analysis has to consider a bidimensional figure of merit. Moreover, for the sake of readability, we choose to represent the $P_{D}$ in terms of contour plots in place of a surf. Thus, given a pair of values $\bar{l}_{1}$ and $\bar{l}_{2}$, we can read the corresponding value of $P_{D}=P_{D}\left(\bar{l}_{1}, \bar{l}_{2}\right)$ for a given value of $P_{\mathrm{FA}}$. Again, we resort to Monte Carlo trials to set the detection thresholds through $100 / P_{\mathrm{FA}}$ independent runs, whereas the $P_{D}$ is estimated over 1000 independent runs.

In Fig. 10, we show the contour plots for the M-PDD-GLRT [see Fig. 10(a)], the GLRT [see Fig. 10(b)], the MLD [see Fig. 10(c)], and the SLD [see Fig. 10(d)]. The figure highlights that, for all the considered decision rules, the $P_{D}$ is lower than 0.1 where both $l_{1}$ and $l_{2}$ are less than $0 \mathrm{~dB}$. However, the detection capability becomes acceptable, increasing at least $l_{1}$ or $l_{2}$. Comparing the aforementioned subplots, it is also clear that the M-PDD-GLRT outperforms the GLRT and the MLD, particularly in areas where a specific eigenvalue is low, and exhibits an overall slightly superior performance than the SLD. Finally, it is worth noting that these curves agree with the behaviors emphasized in the previous analyses; for instance, if we consider the diagonal of the plane $\left(l_{1}, l_{2}\right)$, we can see that the $P_{D}$ values are the same as those shown in Fig. 7 .

Now, we analyze the performance of the proposed detector when there is a mismatch between the numbers of training and test data. This is of paramount importance since, in real environments, the availability of homogeneous data can be very limited. In Fig. 11, we assume that $M=4$ and $K=9$. The trend observed in this figure is quite similar to that in Fig. 10; in fact, the M-PDD-GLRT achieves better detection performances than the GLRT and the MLD for almost all the pairs $\left(l_{1}, l_{2}\right)$, whereas the SLD shares approximately the same $P_{D}$ values as the M-PDD-GLRT.

Finally, we consider the case opposite to the previous one, i.e., the number of training data is less than that of the testing data, i.e., $K=4$ and $M=9$. The corresponding curves are shown in Fig. 12. The M-PDD-GLRT clearly overcomes the

\footnotetext{
${ }^{3}$ Note that $\boldsymbol{R}_{1}=\boldsymbol{I}$.
} 


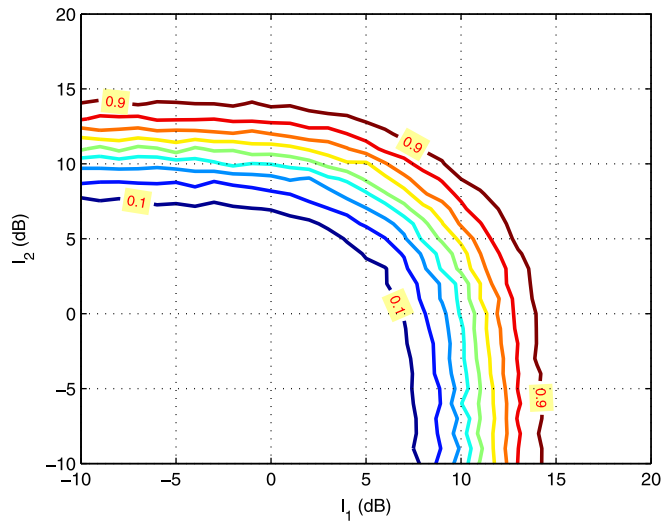

(a)

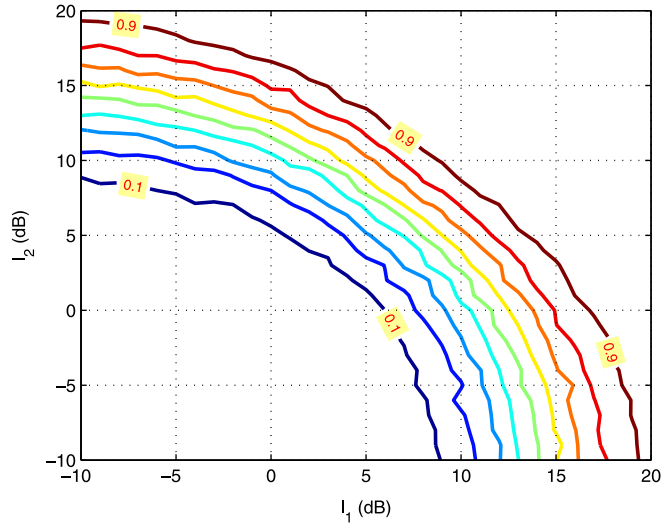

(c)

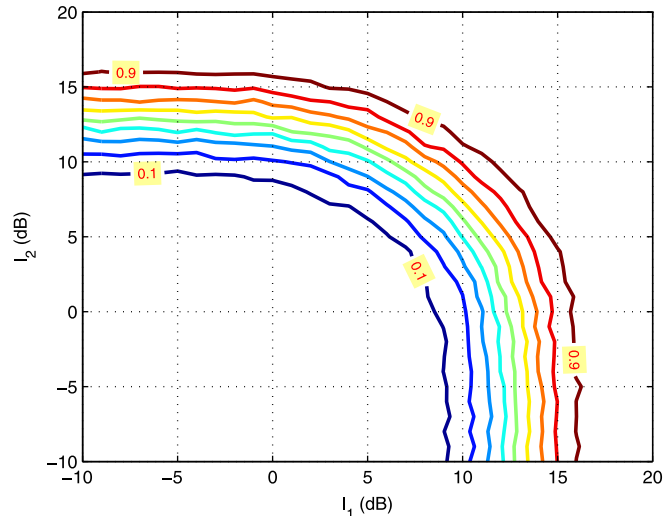

(b)

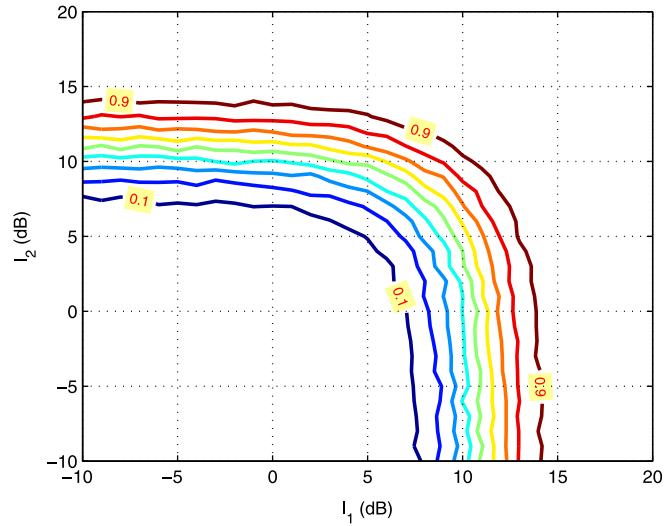

(d)

Fig. 10. $P_{D}$ contours versus $l_{1}$ and $l_{2}$ expressed in decibels for $K=9, M=9$, and $N=3$, for $P_{\mathrm{FA}}=10^{-4}$. (a) M-PDD-GLRT. (b) GLRT. (c) MLD. (d) SLD.

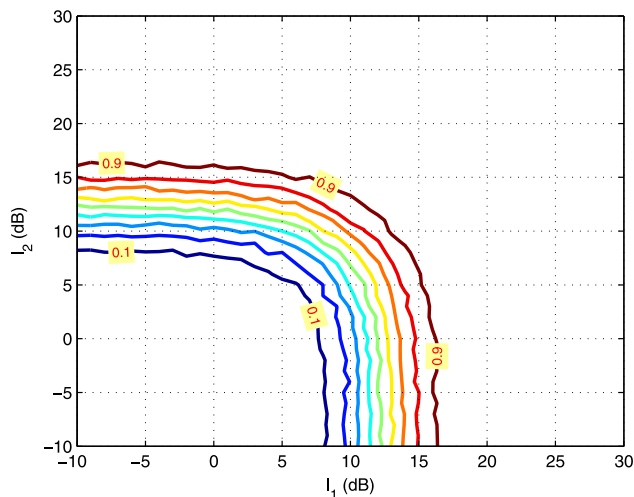

(a)

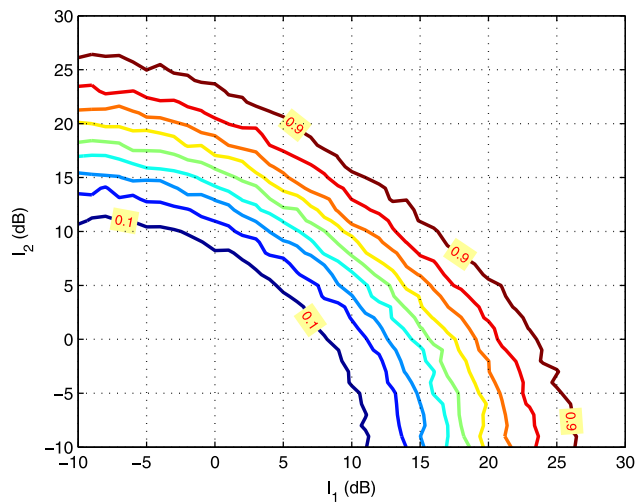

(c)

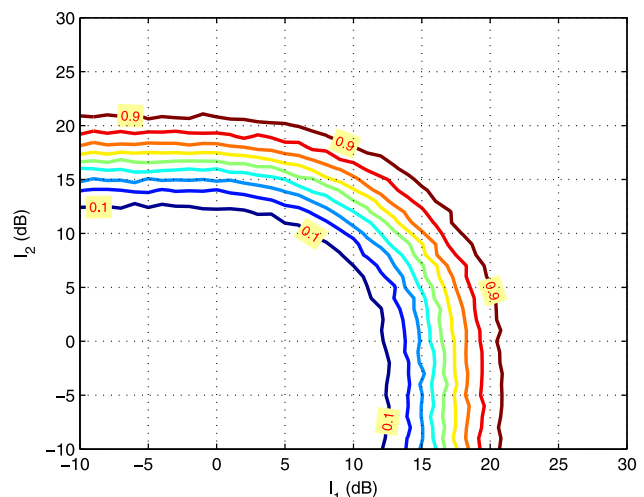

(b)

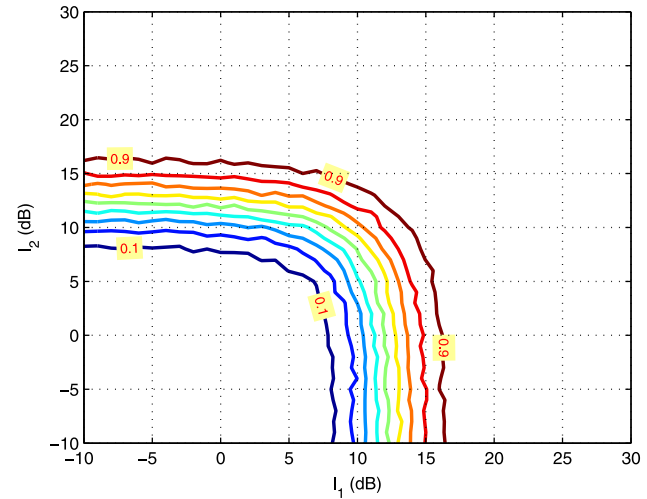

(d)

Fig. 11. $P_{D}$ contours versus $l_{1}$ and $l_{2}$ expressed in decibels for $K=9, M=4$, and $N=3$, for $P_{\mathrm{FA}}=10^{-4}$. (a) M-PDD-GLRT. (b) GLRT. (c) MLD. (d) SLD. 


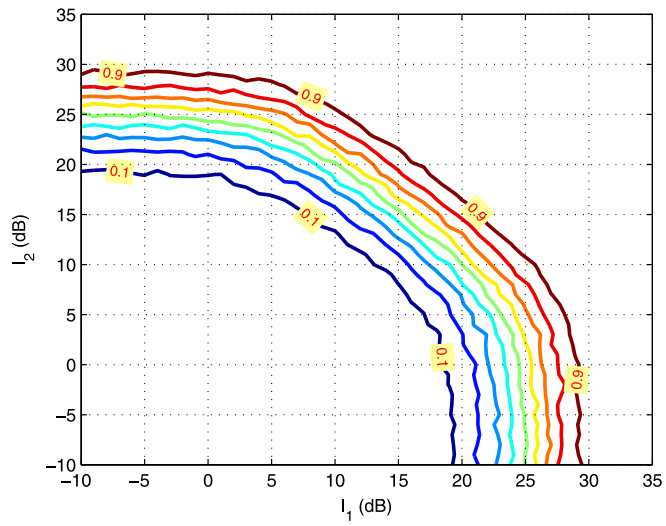

(a)

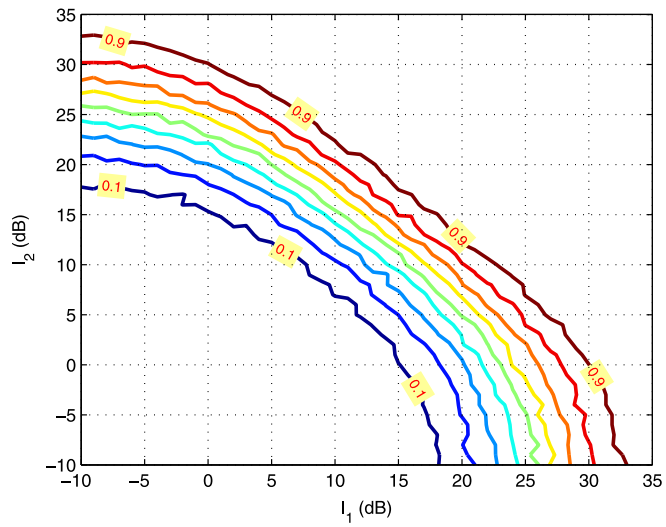

(c)

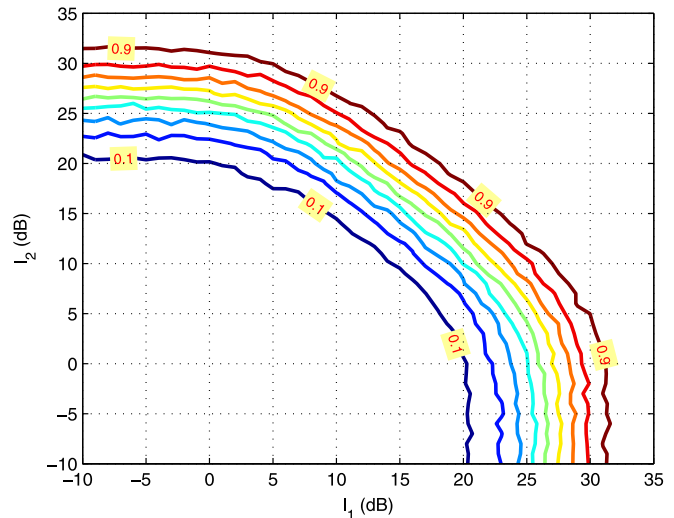

(b)

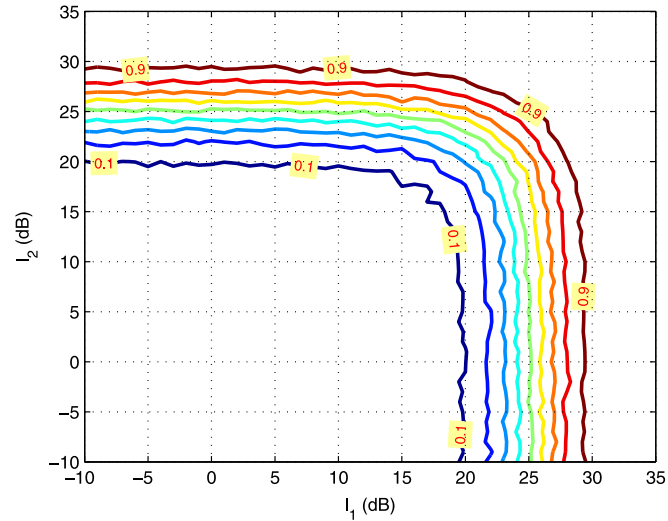

(d)

Fig. 12. $P_{D}$ contours versus $l_{1}$ and $l_{2}$ expressed in decibels for $K=4, M=9$, and $N=3$, for $P_{\text {FA }}=10^{-4}$. (a) M-PDD-GLRT. (b) GLRT. (c) MLD. (d) SLD.

GLRT and the SLD. In particular, the gain over the SLD is much greater in the areas where both the eigenvalues are high. Moreover, comparing the M-PDD-GLRT and the MLD, it is clear that the former is much better in the regions where one eigenvalue is high and the other is low, whereas it exhibits some losses when both eigenvalues are high. For instance, if we consider the pair $l_{1}=-10 \mathrm{~dB}$ and $l_{2}=30 \mathrm{~dB}$, the M-PDD-GLRT exhibits a $P_{D}$ of 0.9 , whereas the MLD achieves that value only when $l_{2}$ is greater than $33 \mathrm{~dB}$. On the contrary, considering the case $l_{1}=10 \mathrm{~dB}$ and $l_{2}=20 \mathrm{~dB}$, we observe that the M-PDD-GLRT reaches the value $P_{D}=0.5$, whereas the MLD ensures a $P_{D}$ of 0.8 .

In a nutshell, this study confirms that the M-PDD-GLRT is less sensitive to parameter variations, ensuring high $P_{D}$ values in all the considered scenarios. Last but not the least, it is able to almost always outperform its counterparts, with acceptable losses when this is not true.

\section{Test on Real Radar Data}

As a final part of the performance analysis, here, we evaluate the effectiveness of the new detectors on real SAR data. More precisely, we use two different data sets concerning the same area obtained from the GOMoil_07601_10052_ 101_100622_L090_CX_02 and GOMoil_07601_10052_102_ 100622_L090_CX_02 SAR images which are composed of sea data containing an oil spill on them. The images have

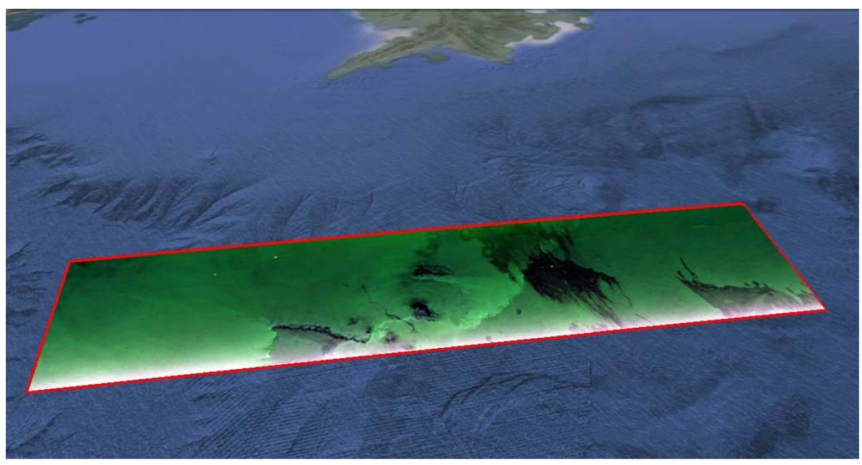

Fig. 13. Three-polarization color overlay of the SAR image GOMoil_07601_ 10052_101_100622_LO90_CX_02.

been acquired on June 22, 2010, during the British Petroleum oil spill incident in the Gulf of Mexico (known also as the Deepwater Horizon Oil Spill), and contain a scene acquired with a sensor exploiting all the polarizations. The corresponding polarimetric overlays are reported in Figs. 13 and 14, respectively.

The first data set is a subimage of $3000 \times 2000$ pixels extracted from acquisition GOMoil_07601_10052_101_ 100622_L090_CX_02, whose span (i.e., $|\mathrm{HH}|^{2}+|\mathrm{VV}|^{2}+$ $2|\mathrm{HV}|^{2}$ ) [16], which is expressed in decibel values, is also displayed in Fig. 15. Fig. 16 shows the detection results applying detector (8) over the described SAR image, where 


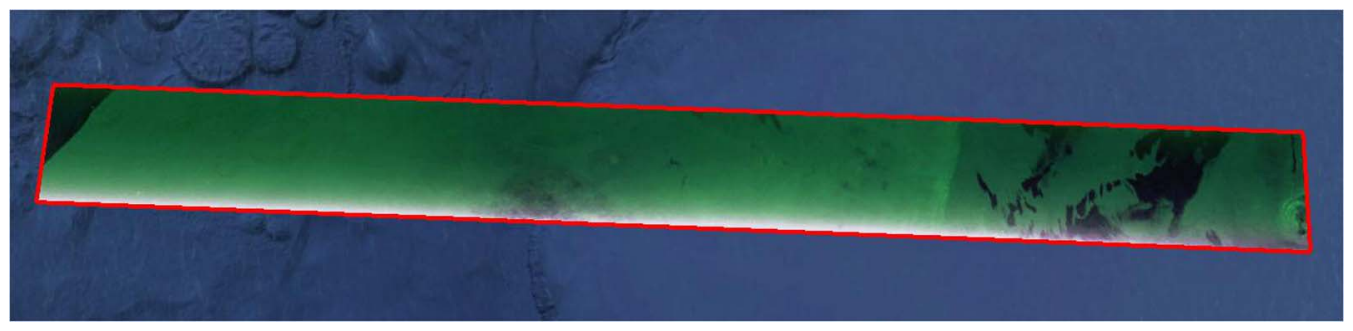

Fig. 14. Three-polarization color overlay of the SAR image GOMoil_07601_10052_102_100622_L090_CX_02.

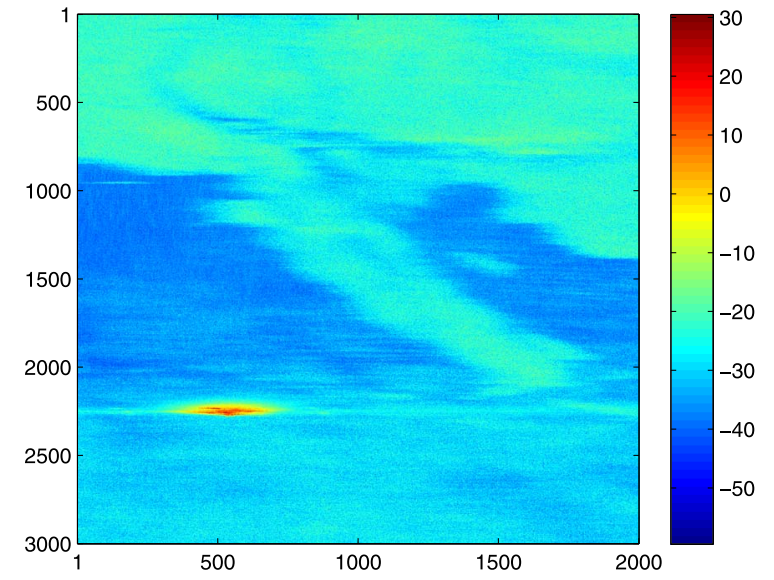

Fig. 15. Real SAR L-band data image GOMoil_07601_10052_101_100622_ L090_CX_02. Span (in decibels) of the reference image of size $3000 \times$ 2000 pixels.

the test is performed assuming the following parameter setting (notice that $N=3$ since we have three polarization images). A sea data pixel is chosen as a reference to compute the sample covariance $\boldsymbol{H}$ from a $3 \times 3$ (i.e., $M=9$ ) window centered in that pixel and from a $4 \times 1$ slice of pixels (i.e., $M=4$ ) with the considered pixel in position $(2,1)$, respectively. Then, a window of size $3 \times 3$ (i.e., $K=9$ ) is slided over the SAR image to test all the pixels and to compute the sample matrix $\boldsymbol{G}$. Finally, the threshold is chosen to ensure a nominal $P_{\mathrm{FA}}$ of $10^{-3}$ (and $10^{-4}$ ), extracting a cluster of only sea data composed of $10^{5}$ (and $10^{6}$ ) pixels and choosing the 100th value in the decreasing ordered statistics. The result is a detection map where the white pixels are those associated to oil spill detections, whereas the black pixels are representative of the sea data (no detections).

For comparison purposes, Fig. 17 contains the detection performances that come from the application of a threshold to the span image. Again, $P_{\mathrm{FA}}$ is set to $10^{-3}$.

The results show that the multifamily PDD is able to ensure reliable detection of oil spills over the sea with less false alarms than those produced by thresholding the span image.

Additionally, in Fig. 16, it can be observed that the exploitation of a great number of testing data ensures better detection performance, even if, in real environment, this number has to be chosen to manage the tradeoff between the accuracy of covariance estimation and the amount of heterogeneity.

Finally, in Fig. 18, the receiver operating characteristic (ROC) curves for the M-PDD-GLRT and the SLD are plotted using the real SAR image. The latter architecture has been chosen as a basis for comparison because its performances are close to those of the M-PDD-GLRT in simulated scenarios. The curves are obtained setting the thresholds that ensure the nominal $P_{\mathrm{FA}}$ through the rule $100 / P_{\mathrm{FA}}$, exploiting data from sea-only pixels, and the corresponding $P_{D}$ are then evaluated, focusing on a zone containing the oil spills.

It turns out that the M-PDD-GLRT exhibits a detection gain over the SLD greater than or equal to 0.1 for $P_{\mathrm{FA}} \leq 10^{-2}$.

The second data set is obtained from the acquisition $G O$ Moil_07601_10052_102_100622_L090_CX_02. Specifically, a subimage of $9749 \times 9898$ pixels is considered, whose span (expressed in decibels) is displayed in Fig. 19. Fig. 20 shows the detection results of the M-PDD-GLRT over this second subimage, whereas Fig. 21 is analogous to Fig. 17, i.e., it contains the detection map obtained thresholding the span image. The parameter setting and the procedure to compute the detection threshold are the same as in Fig. 16. Again, the white pixels are associated to the detections of oil spills over the sea, whereas the black pixels are representative of the sea data (no detections).

As already shown for the first data set, the results confirm the ability of the M-PDD-GLRT to correctly detect the oil spills over the sea with a lower number of false alarms than the heuristic method based on the span image thresholding. Finally, the ROC curves for the M-PDD-GLRT and the SLD are plotted in Fig. 22. The hierarchy observed in the previous illustrative examples is confirmed, except for Fig. 22(a), where the considered architectures share almost the same performance.

\section{CONCLUSION}

Multipolarization SAR detection of oil spills has been considered in this paper to test the equality of two polarimetric sample covariance matrices constructed from a reference area (where the absence of oil slicks is known) and a test region (where it is necessary to establish the spot presence), respectively. The alternative hypothesis is represented by the instance where the PCM within the reference area exhibits at least an ordered eigenvalue greater than the corresponding one extracted from the PCM of the area under test. First of all, assuming the exact knowledge about the number of different eigenvalues between the reference and tested PCMs, the PDD-GLRT is devised. Then, to come up with a fully adaptive detector without any a priori assumption about the aforementioned number, the M-PDD-GLRT is introduced discussing invariance and CFAR properties. 


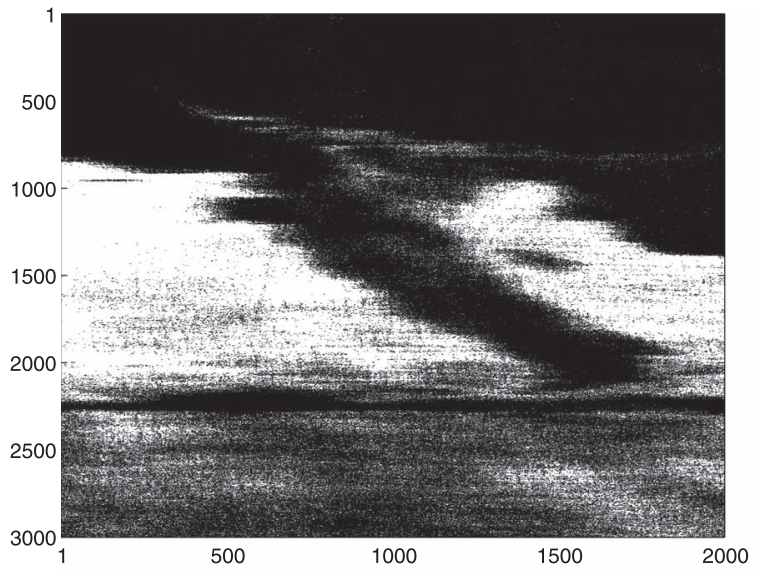

(a)

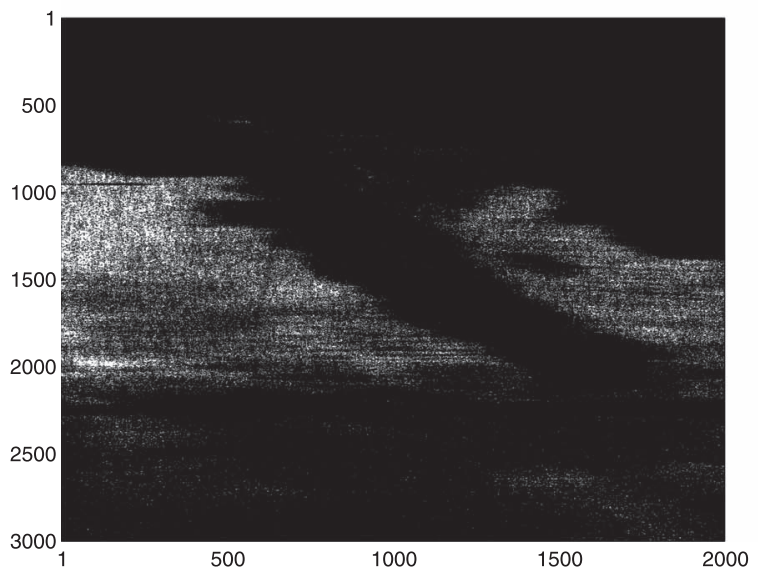

(c)

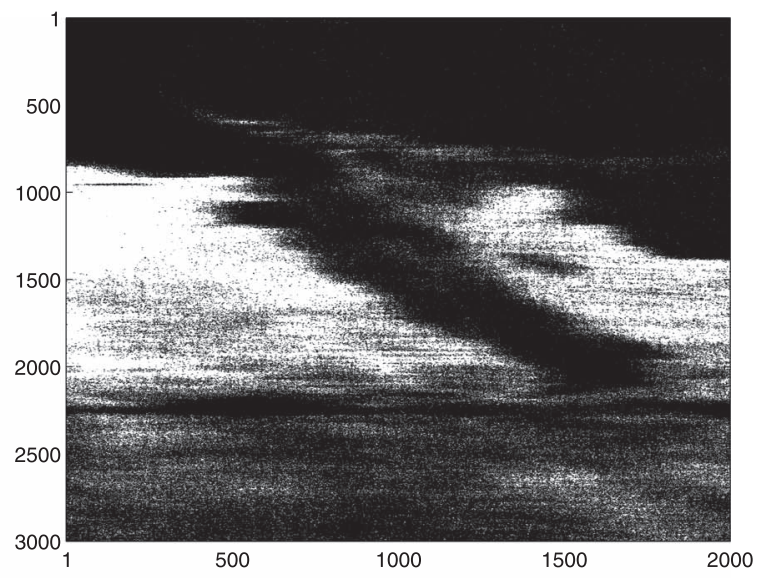

(b)

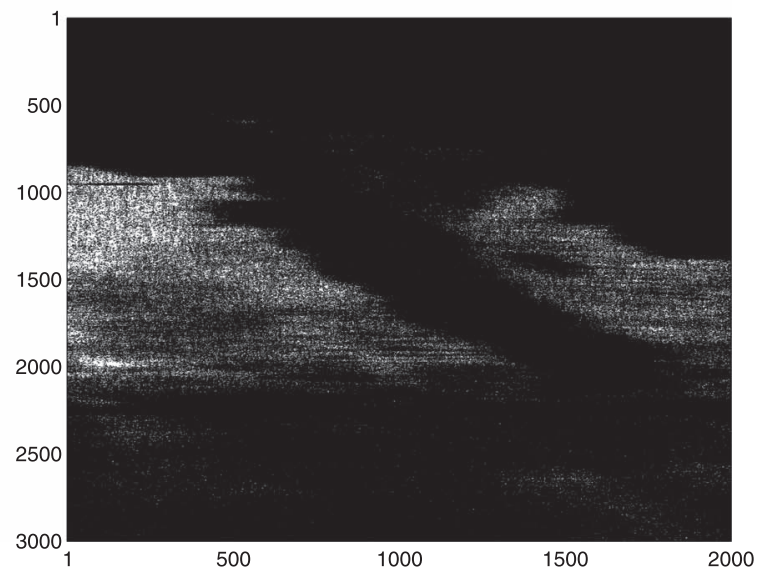

(d)

Fig. 16. Real SAR L-band data SAR GOMoil_07601_10052_101_100622_LO90_CX_02 detection map for the M-PDD-GLRT with $K=9$ and $N=3$. The detection map has been obtained with a nominal (a) and (b) $P_{\mathrm{FA}}=10^{-3}$ and (c) and (d) $P_{\mathrm{FA}}=10^{-4}$. The white pixels are the detections. Subplots (a) and (c) refer to $M=9$, whereas (b) and (d) refer to $M=4$. (a) $K=9, M=9, P_{\mathrm{FA}}=10^{-3}$. (b) $K=9, M=4, P_{\mathrm{FA}}=10^{-3}$. (c) $K=9, M=9, P_{\mathrm{FA}}=10^{-4}$. (d) $K=9, M=4, P_{\mathrm{FA}}=10^{-4}$.

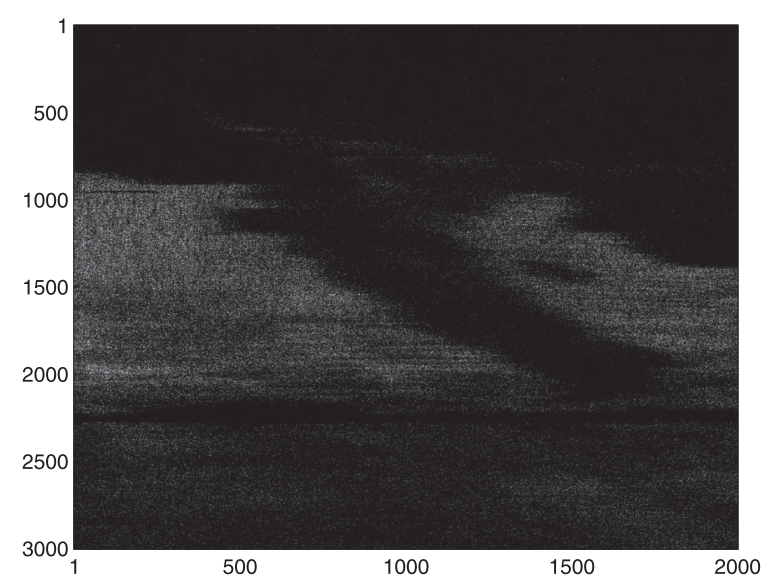

Fig. 17. Real SAR L-band data SAR GOMoil_07601_10052_101_100622_ L090_CX_02 detection map resulting from the application of a threshold to the span image. The detection map has been obtained with a nominal $P_{\mathrm{FA}}=10^{-3}$. The white pixels are the detections.

At the analysis stage, examples have been provided to prove the effectiveness of the proposed approach also in comparison with existing strategies for oil spill detection. To this end, both simulated and real multipolarization SAR data have been used. Possible future research track might concern the extension of the approach to the case of a joint multifrequency and multipolarization processing and to consider the presence of non-Gaussian backscattering due to the sea.

\section{APPENDIX I \\ DERIVATION OF THE PDD-GLRT}

Let us begin focusing on the maximization problem under $H_{1}$ and consider the log likelihood, which, neglecting constants, is

$$
\begin{aligned}
\log [ & \left.f\left(\boldsymbol{Z}_{K}, \boldsymbol{Y}_{M} ; \boldsymbol{R}_{1}, \boldsymbol{R}_{2}, H_{1}\right)\right] \\
\approx & -K \log \left[\operatorname{det}\left(\boldsymbol{R}_{1}\right)\right]-M \log \left[\operatorname{det}\left(\boldsymbol{R}_{1}+\boldsymbol{R}_{2}\right)\right] \\
& -\sum_{i=1}^{K} \boldsymbol{z}_{i}^{\dagger} \boldsymbol{R}_{1}^{-1} \boldsymbol{z}_{i}-\sum_{j=1}^{M} \boldsymbol{y}_{j}^{\dagger}\left(\boldsymbol{R}_{1}+\boldsymbol{R}_{2}\right)^{-1} \boldsymbol{y}_{j} .
\end{aligned}
$$

Now, denote by $\lambda_{1} \geq \lambda_{2} \geq \cdots \geq \lambda_{p}>\lambda_{p+1}=\cdots=\lambda_{N}=1$ the roots of

$$
\operatorname{det}\left[\left(\boldsymbol{R}_{1}+\boldsymbol{R}_{2}\right)-\lambda \boldsymbol{R}_{1}\right]=0
$$

where we recall that $p$ is the rank of $\boldsymbol{R}_{2}$, and define

$$
\boldsymbol{A}=\boldsymbol{R}_{1}^{\frac{1}{2}} \boldsymbol{U}
$$




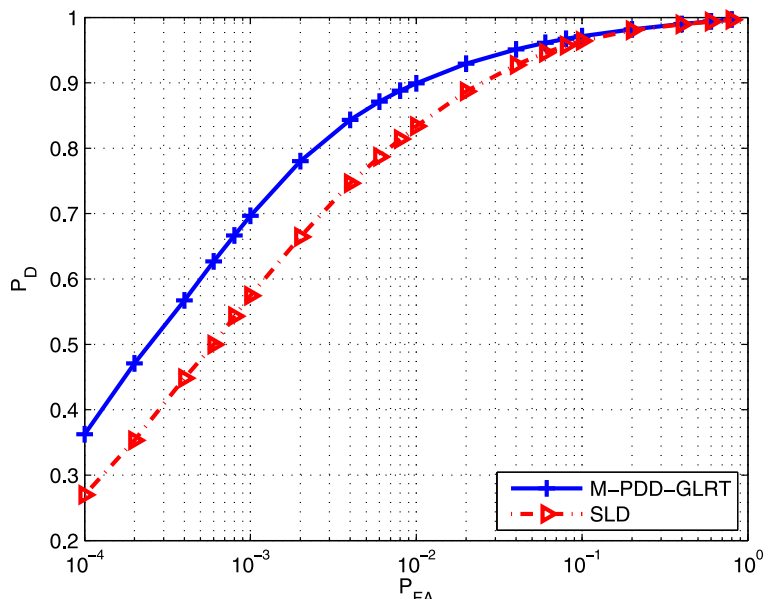

(a)

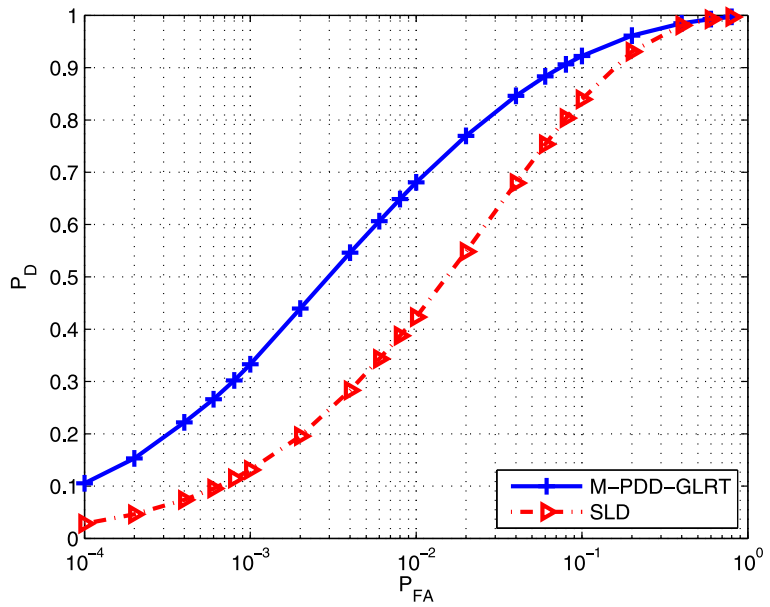

(b)

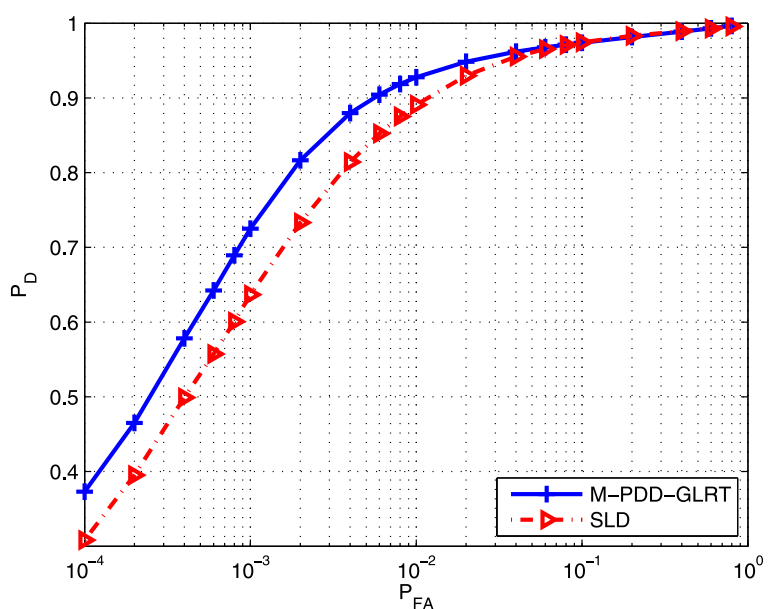

(c)

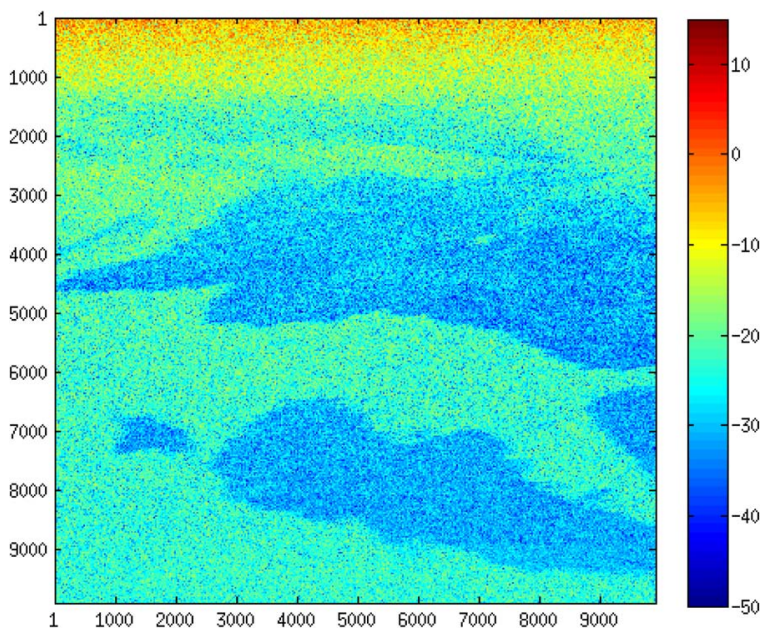

Fig. 19. Real SAR L-band data GOMoil_07601_10052_102_100622_L090_ CX_02. Span (in decibels) of the reference image of size $9749 \times 9898$ pixels.

that is

$$
\boldsymbol{C}=\boldsymbol{U} \boldsymbol{\Lambda} \boldsymbol{U}^{\dagger}
$$

with $\Lambda$ being a diagonal matrix whose entries are the eigenvalues $^{4}$ of $\boldsymbol{C}$, i.e.,

$$
\boldsymbol{\Lambda}=\left[\begin{array}{cccccc}
\lambda_{1} & 0 & & \cdots & & 0 \\
0 & \ddots & & & & \\
& & \lambda_{p} & \ddots & & \vdots \\
\vdots & & \ddots & 1 & & \\
& & & & \ddots & 0 \\
0 & & \ldots & & 0 & 1
\end{array}\right] .
$$

It follows that

$$
\boldsymbol{R}_{1}=\boldsymbol{A} \boldsymbol{A}^{\dagger} \quad\left(\boldsymbol{R}_{1}+\boldsymbol{R}_{2}\right)=\boldsymbol{A} \boldsymbol{\Lambda} \boldsymbol{A}^{\dagger}
$$

and the right-hand side of (24) can be recast as

$$
\begin{aligned}
(24)= & -2 K \log \operatorname{det}(\boldsymbol{A})-2 M \log \operatorname{det}(\boldsymbol{A})-M \log \operatorname{det}(\boldsymbol{\Lambda}) \\
& -\operatorname{tr}\left\{\left(\boldsymbol{A} \boldsymbol{A}^{\dagger}\right)^{-1} \boldsymbol{G}-\left(\boldsymbol{A} \boldsymbol{\Lambda} \boldsymbol{A}^{\dagger}\right)^{-1} \boldsymbol{H}\right\} \\
= & -a \log \operatorname{det}(\boldsymbol{A})-M \log \operatorname{det}(\boldsymbol{\Lambda}) \\
& -\operatorname{tr}\left\{\left(\boldsymbol{A}^{\dagger}\right)^{-1} \boldsymbol{A}^{-1} \boldsymbol{G}\right\}-\operatorname{tr}\left\{\left(\boldsymbol{A}^{\dagger}\right)^{-1} \boldsymbol{\Lambda}^{-1} \boldsymbol{A}^{-1} \boldsymbol{H}\right\}
\end{aligned}
$$

where $a=2 K+2 M$.

Now, denote by $\delta_{1}>\delta_{2}>\cdots>\delta_{N}>0$ the roots of

$$
\operatorname{det}(\boldsymbol{H}-\delta \boldsymbol{G})=0
$$

which are the eigenvalues of $\boldsymbol{G}^{-1 / 2} \boldsymbol{H} \boldsymbol{G}^{-1 / 2}$, whose eigendecomposition is given by

$$
\boldsymbol{U}_{\Delta} \boldsymbol{\Delta} \boldsymbol{U}_{\Delta}^{\dagger}
$$

$$
\boldsymbol{C}=\boldsymbol{R}_{1}^{-1 / 2}\left(\boldsymbol{R}_{1}+\boldsymbol{R}_{2}\right) \boldsymbol{R}_{1}^{-1 / 2}
$$

${ }^{4}$ Note that $\boldsymbol{C}$ and $\left(\boldsymbol{I}+\boldsymbol{R}_{1}^{-1} \boldsymbol{R}_{2}\right)$ share the same eigenvalues. 


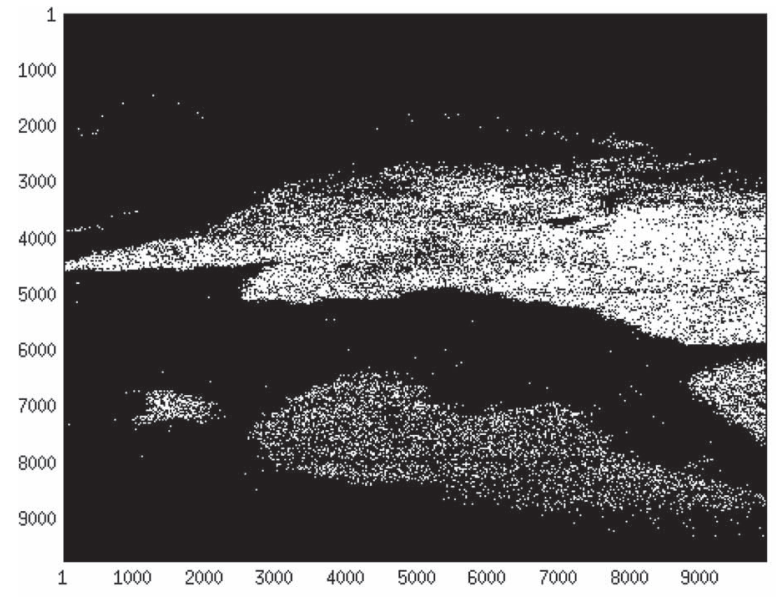

(a)

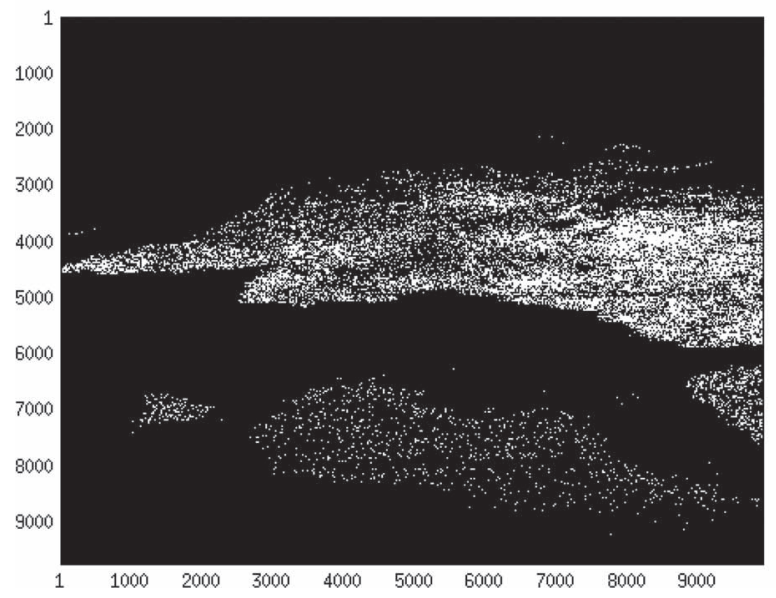

(c)

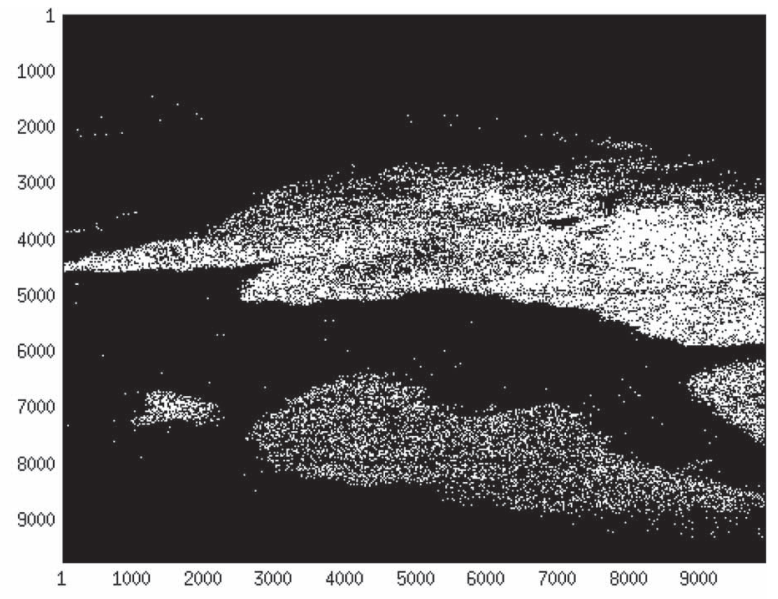

(b)

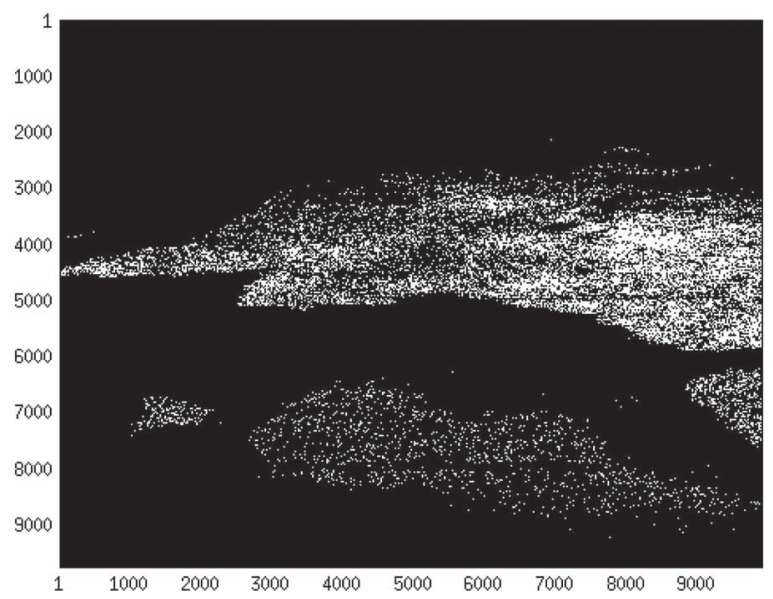

(d)

Fig. 20. Real SAR L-band data GOMoil_07601_10052_102_100622_L090_CX_02 detection map for the M-PDD-GLRT with $K=9$ and $N=3$. The detection map has been obtained with a nominal (a) and (b) $P_{\mathrm{FA}}=10^{-3}$ and (c) and (d) $P_{\mathrm{FA}}=10^{-4}$. The white pixels are the detections. Subplots (a) and (c) refer to $M=9$, whereas (b) and (d) refer to $M=4$. (a) $K=9, M=9, P_{\mathrm{FA}}=10^{-3}$, (b) $K=9, M=4, P_{\mathrm{FA}}=10^{-3}$. (c) $K=9, M=9, P_{\mathrm{FA}}=10^{-4}$. (d) $K=9, M=4, P_{\mathrm{FA}}=10^{-4}$.

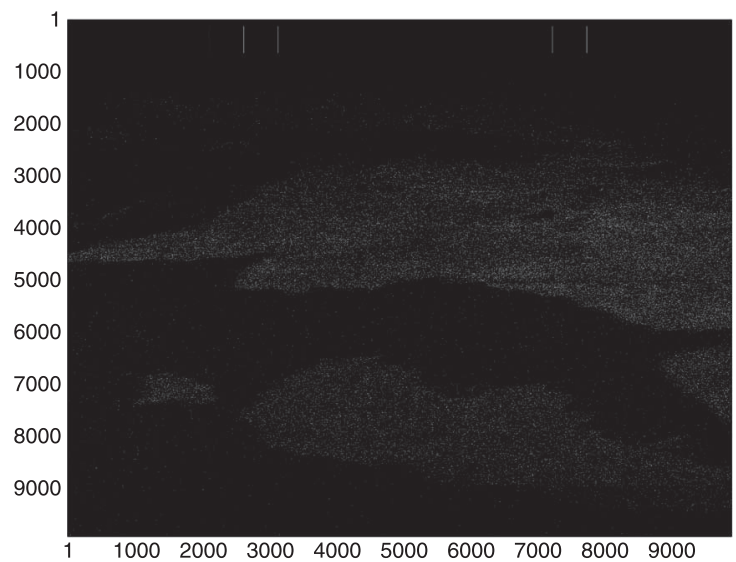

Fig. 21. Real SAR L-band data GOMoil_07601_10052_102_100622_L090_ CX_02 detection map resulting from the application of a threshold to the span image. The detection map has been obtained with a nominal $P_{\mathrm{FA}}=10^{-3}$. The white pixels are the detections.

where

$$
\boldsymbol{\Delta}=\left[\begin{array}{ccc}
\delta_{1} & & 0 \\
& \ddots & \\
0 & & \delta_{N}
\end{array}\right]
$$

and $U_{\Delta}$ is the matrix whose columns are the corresponding normalized eigenvectors, i.e., $\boldsymbol{U}_{\Delta} \boldsymbol{U}_{\Delta}^{\dagger}=\boldsymbol{U}_{\Delta}^{\dagger} \boldsymbol{U}_{\Delta}=\boldsymbol{I}$. Again, define a further matrix $\boldsymbol{B}=\boldsymbol{G}^{1 / 2} \boldsymbol{U}_{\Delta}$ and observe that

$$
\begin{aligned}
& \boldsymbol{G}=\boldsymbol{B} \boldsymbol{B}^{\dagger} \\
& \boldsymbol{H}=\boldsymbol{B} \boldsymbol{\Delta} \boldsymbol{B}^{\dagger} .
\end{aligned}
$$

As a consequence, (31) can be written as

$$
\begin{aligned}
(31)= & -a \log \operatorname{det}(\boldsymbol{A})-M \log \operatorname{det}(\boldsymbol{\Lambda}) \\
& -\operatorname{tr}\left\{\left(\boldsymbol{A}^{\dagger}\right)^{-1} \boldsymbol{A}^{-1} \boldsymbol{B} \boldsymbol{B}^{\dagger}\right\} \\
& -\operatorname{tr}\left\{\left(\boldsymbol{A}^{\dagger}\right)^{-1} \boldsymbol{\Lambda}^{-1} \boldsymbol{A}^{-1} \boldsymbol{B} \boldsymbol{\Delta} \boldsymbol{B}^{\dagger}\right\} \\
= & -a \log \operatorname{det}(\boldsymbol{A})-M \log \operatorname{det}(\boldsymbol{\Lambda}) \\
& -\operatorname{tr}\left\{\boldsymbol{A}^{-1} \boldsymbol{B} \boldsymbol{\Delta}^{\frac{1}{2}} \boldsymbol{\Delta}^{-1} \boldsymbol{\Delta}^{\frac{1}{2}} \boldsymbol{B}^{\dagger}\left(\boldsymbol{A}^{-1}\right)^{\dagger}\right\} \\
& -\operatorname{tr}\left\{\boldsymbol{\Lambda}^{-1} \boldsymbol{A}^{-1} \boldsymbol{B} \boldsymbol{\Delta}^{\frac{1}{2}} \boldsymbol{\Delta}^{\frac{1}{2}} \boldsymbol{B}^{\dagger}\left(\boldsymbol{A}^{-1}\right)^{\dagger}\right\} .
\end{aligned}
$$

Now, observe that problem

$$
\max _{\boldsymbol{R}_{1}} \max _{\boldsymbol{R}_{2}} f\left(\boldsymbol{Z}_{K}, \boldsymbol{Y}_{M} ; \boldsymbol{R}_{1}, \boldsymbol{R}_{2}, H_{1}\right)
$$




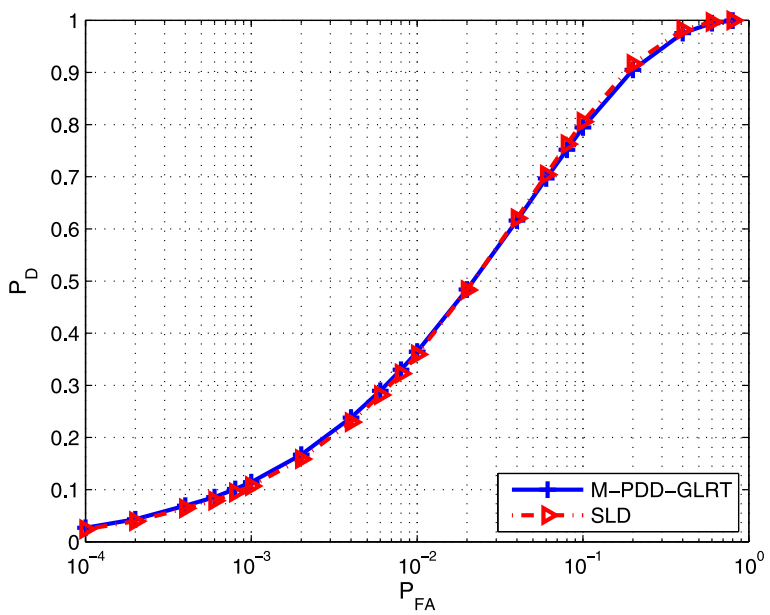

(a)

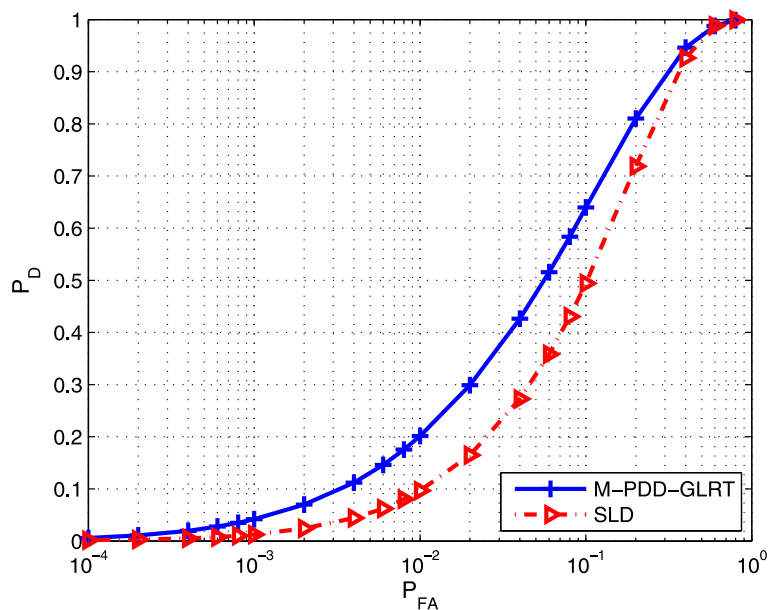

(b)

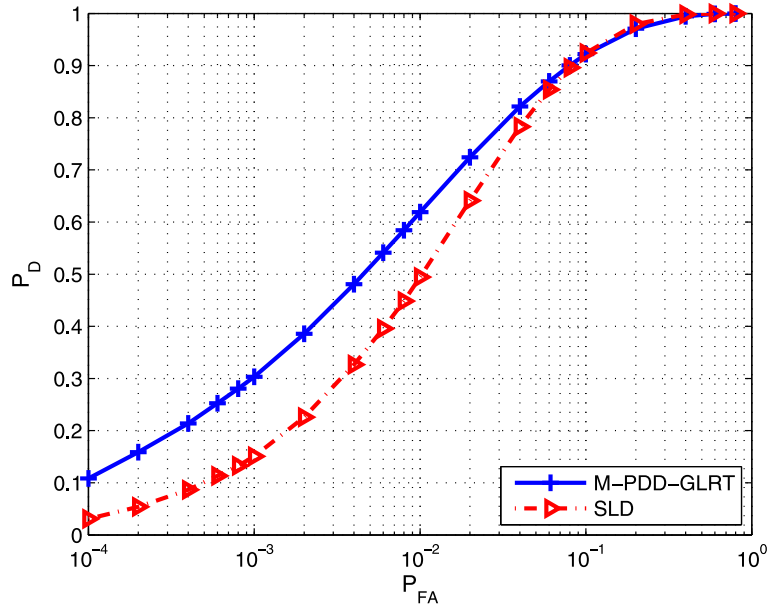

(c)

Fig. 22. $P_{D}$ versus $P_{\mathrm{FA}}$ for the M-PDD-GLRT and the SLD. Real SAR L-band data GOMoil_07601_10052_102_100622_L090_CX_02. (a) $K=9$, $M=4, P_{\mathrm{FA}}=10^{-\overline{3}}$. (b) $\bar{K}=4, \bar{M}=9, P_{\mathrm{FA}}=10^{-3}$. (c) $K=9, M=$ $9, P_{\mathrm{FA}}=10^{-4}$.

is tantamount to maximizing (31) over $\boldsymbol{\Lambda}, \boldsymbol{\Delta}$, and $\boldsymbol{A}$. To this end, we exploit the singular value decomposition of matrix $\boldsymbol{A}^{-1} \boldsymbol{B} \boldsymbol{\Delta}^{1 / 2}$, i.e.,

$$
\boldsymbol{A}^{-1} \boldsymbol{B} \boldsymbol{\Delta}^{\frac{1}{2}}=\boldsymbol{P} \boldsymbol{D}_{r} \boldsymbol{Q}
$$

where $\boldsymbol{P}, \boldsymbol{Q} \in \mathbb{C}^{N \times N}$ are unitary matrices, and

$$
\boldsymbol{\Delta}_{r}=\left[\begin{array}{ccc}
r_{1} & & 0 \\
& \ddots & \\
0 & & r_{N}
\end{array}\right]
$$

with $r_{1} \geq \cdots \geq r_{N}>0$. From (38), it is possible to write $\boldsymbol{A}^{-1}$ and its determinant as follows:

$$
\begin{aligned}
\boldsymbol{A}^{-1} & =\boldsymbol{P} \boldsymbol{D}_{r} \boldsymbol{Q} \boldsymbol{\Delta}^{-1 / 2} \boldsymbol{B}^{-1} \\
\operatorname{det}\left(\boldsymbol{A}^{-1}\right) & =\operatorname{det}(\boldsymbol{A})^{-1}=\operatorname{det}\left(\boldsymbol{D}_{r}\right) \operatorname{det}(\boldsymbol{\Delta})^{-1 / 2} \operatorname{det}(\boldsymbol{B})^{-1} \\
& =\operatorname{det}\left(\boldsymbol{D}_{r}\right) \operatorname{det}(\boldsymbol{B})^{-1} \operatorname{det}(\boldsymbol{\Delta})^{-1 / 2}
\end{aligned}
$$

The latter equations can be exploited to recast (36) as

$$
\begin{aligned}
(36)= & a\left[\log \operatorname{det}\left(\boldsymbol{D}_{r}\right)+\log \operatorname{det}(\boldsymbol{B})^{-1}+\log \operatorname{det}(\boldsymbol{\Delta})^{-1 / 2}\right] \\
& -M \log \operatorname{det}(\boldsymbol{\Lambda})-\operatorname{tr}\left\{\boldsymbol{P} \boldsymbol{D}_{r} \boldsymbol{Q} \boldsymbol{\Delta}^{-1}\left(\boldsymbol{P} \boldsymbol{D}_{r} \boldsymbol{Q}\right)^{\dagger}\right\} \\
& -\operatorname{tr}\left\{\boldsymbol{\Lambda}^{-1} \boldsymbol{P} \boldsymbol{D}_{r} \boldsymbol{Q}\left(\boldsymbol{P} \boldsymbol{D}_{r} \boldsymbol{Q}\right)^{\dagger}\right\} \\
= & a \log \operatorname{det}\left(\boldsymbol{D}_{r}\right)-a \log \operatorname{det}(\boldsymbol{B})-\frac{a}{2} \log \operatorname{det}(\boldsymbol{\Delta}) \\
& -M \log \operatorname{det}(\boldsymbol{\Lambda})-\operatorname{tr}\left\{\boldsymbol{D}_{r}^{2} \boldsymbol{Q} \boldsymbol{\Delta}^{-1} \boldsymbol{Q}^{\dagger}\right\} \\
& -\operatorname{tr}\left\{\boldsymbol{\Lambda}^{-1} \boldsymbol{P} \boldsymbol{D}_{r}^{2} \boldsymbol{P}^{\dagger}\right\} .
\end{aligned}
$$

It is clear that problem (37) can be solved maximizing (42) with respect to $\boldsymbol{\Lambda}, \boldsymbol{P}, \boldsymbol{Q}$, and $\boldsymbol{D}_{r}$. Thus, let us focus on the following function:

$$
\begin{aligned}
g\left(\boldsymbol{D}_{r}, \boldsymbol{\Lambda}, \boldsymbol{P}, \boldsymbol{Q}\right)= & a \log \operatorname{det}\left(\boldsymbol{D}_{r}\right)-M \log \operatorname{det}(\boldsymbol{\Lambda}) \\
& -\operatorname{tr}\left\{\boldsymbol{D}_{r}^{2} \boldsymbol{Q} \boldsymbol{\Delta}^{-1} \boldsymbol{Q}^{\dagger}\right\} \\
& -\operatorname{tr}\left\{\boldsymbol{\Lambda}^{-1} \boldsymbol{P} \boldsymbol{D}_{r}^{2} \boldsymbol{P}^{\dagger}\right\} .
\end{aligned}
$$

Maximization over $\boldsymbol{P}$ and $\boldsymbol{Q}$ can be performed exploiting Theorem 1 of $^{5}$ [22]. More precisely, it is not difficult to show that

$$
\begin{aligned}
& \max _{\boldsymbol{P}} \max _{\boldsymbol{Q}} g\left(\boldsymbol{D}_{r}, \boldsymbol{\Lambda}, \boldsymbol{P}, \boldsymbol{Q}\right) \\
&= a \log \operatorname{det}\left(\boldsymbol{D}_{r}\right)-M \log \operatorname{det}(\boldsymbol{\Lambda}) \\
&-\operatorname{tr}\left\{\boldsymbol{D}_{r}^{2} \boldsymbol{\Delta}^{-1}\right\}-\operatorname{tr}\left\{\boldsymbol{\Lambda}^{-1} \boldsymbol{D}_{r}^{2}\right\} \\
&= a \sum_{i=1}^{N} \log r_{i}-M \sum_{i=1}^{N} \log \lambda_{i}-\sum_{i=1}^{N} \frac{r_{i}^{2}}{\delta_{i}}-\sum_{i=1}^{N} \frac{r_{i}^{2}}{\lambda_{i}} \\
&= \sum_{i=1}^{N}\left\{\frac{a}{2} \log r_{i}^{2}-M \log \lambda_{i}-r_{i}^{2}\left(\frac{1}{\delta_{i}}+\frac{1}{\lambda_{i}}\right)\right\} \\
&= g\left(\boldsymbol{D}_{r}, \boldsymbol{\Lambda}, \hat{\boldsymbol{P}}, \hat{\boldsymbol{Q}}\right)
\end{aligned}
$$

${ }^{5}$ For the reader' convenience, we provide here the theorem statement. More precisely, if $\boldsymbol{K}$ and $\boldsymbol{L}$ are Hermitian $N$-dimensional matrices with eigenvalues $x_{1} \geq \cdots, \geq x_{N}$ and $\lambda_{1} \geq \cdots, \geq \lambda_{N}$, respectively, then

$$
\sum_{r=1}^{N} x_{r} \lambda_{N-r+1} \leq \operatorname{Tr}[\boldsymbol{K} \boldsymbol{L}] \leq \sum_{r=1}^{N} x_{r} \lambda_{r} .
$$


where

$$
\begin{aligned}
\hat{\boldsymbol{P}} & =\arg \max _{\boldsymbol{P}} g\left(\boldsymbol{D}_{r}, \boldsymbol{\Lambda}, \boldsymbol{P}, \boldsymbol{Q}\right)=\hat{\boldsymbol{Q}} \\
& =\arg \max _{\boldsymbol{Q}} g\left(\boldsymbol{D}_{r}, \boldsymbol{\Lambda}, \boldsymbol{P}, \boldsymbol{Q}\right)=\boldsymbol{I} .
\end{aligned}
$$

As to the optimization over $\boldsymbol{D}_{r}$, it can be accomplished solving the following system of equations ${ }^{6}$ :

$$
\frac{\partial}{\partial y_{i}} g\left(\boldsymbol{D}_{r}, \boldsymbol{\Lambda}, \hat{\boldsymbol{P}}, \hat{\boldsymbol{Q}}\right)=0, \quad i=0, \ldots, N
$$

where $y_{i}=r_{i}^{2}$. It is not difficult to show that the solution of the preceding system is

$$
\hat{y}_{i}=\frac{a}{2} \frac{1}{\left(\frac{1}{\delta_{i}}+\frac{1}{\lambda_{i}}\right)}, \quad i=1, \ldots, N .
$$

Substituting the right-hand side of (47) in place of $r_{i}^{2}, i=$ $1, \ldots, N$, in (44) leads to

$$
\begin{array}{r}
g\left(\hat{\boldsymbol{D}}_{r}, \boldsymbol{\Lambda}, \hat{\boldsymbol{P}}, \hat{\boldsymbol{Q}}\right)=\sum_{i=1}^{N}\left\{\frac{a}{2} \log \frac{a}{2}-\frac{a}{2} \log \left(\frac{1}{\delta_{i}}+\frac{1}{\lambda_{i}}\right)\right. \\
\left.-\frac{a}{2}-M \log \lambda_{i}\right\} .
\end{array}
$$

Finally, it still remains to maximize $g\left(\hat{\boldsymbol{D}}_{r}, \boldsymbol{\Lambda}, \hat{\boldsymbol{P}}, \hat{\boldsymbol{Q}}\right)$ with respect to $\boldsymbol{\Lambda}$. To this end, we set to zero the first derivative of $g\left(\hat{\boldsymbol{D}}_{r}, \boldsymbol{\Lambda}, \hat{\boldsymbol{P}}, \hat{\boldsymbol{Q}}\right)$ with respect to $\lambda_{i}, i=1, \ldots, N$, to obtain the following system of equations:

$$
\begin{aligned}
\frac{\partial}{\partial \lambda_{i}} & g\left(\hat{\boldsymbol{D}}_{r}, \boldsymbol{\Lambda}, \hat{\boldsymbol{P}}, \hat{\boldsymbol{Q}}\right) \\
& =\frac{\partial}{\partial \lambda_{i}}\left\{\frac{a}{2} \log \frac{a}{2}-\frac{a}{2}-\frac{a}{2} \log \left(\frac{1}{\delta_{i}}+\frac{1}{\lambda_{i}}\right)-M \log \lambda_{i}\right\} \\
& =\frac{\partial}{\partial \lambda_{i}}\left\{a_{1}-\frac{a}{2} \log \left(\frac{1}{\delta_{i}}+\frac{1}{\lambda_{i}}\right)-M \log \lambda_{i}\right\} \\
& =\frac{a}{2} \frac{1}{\frac{1}{\delta_{i}}+\frac{1}{\lambda_{i}}} \frac{1}{\lambda_{i}^{2}}-\frac{M}{\lambda_{i}}=0, \quad i=1, \ldots, N
\end{aligned}
$$

Its solution is

$$
\hat{\lambda}_{i}=\delta_{i}\left(\frac{\frac{a}{2}-M}{M}\right)=\delta_{i} \frac{K}{M}, \quad i=1, \ldots, N
$$

if $\delta_{i}>(M / K)$; when the latter condition is not satisfied, then $\hat{\lambda}_{i}=1$.

Now, let $m^{*}$ be the number of $\delta_{i}>M / K$, which represents an estimate of the rank of $\boldsymbol{R}_{2}$, and define

$$
p^{*}=\min \left\{p, m^{*}\right\} .
$$

Since the rank of $\boldsymbol{R}_{2}$ is known, if $p^{*}<p$, then we set $\Lambda\left(\boldsymbol{Z}_{K}, \boldsymbol{Y}_{M}\right)=1$; on the other hand, we can continue the

${ }^{6}$ Recall that $r_{i}>0, \forall i$ derivation of the GLRT evaluating function $g(\cdot, \cdot, \cdot, \cdot)$ at the ML estimates of the arguments, i.e.,

$$
\begin{aligned}
& g\left(\hat{\boldsymbol{D}}_{r}, \hat{\boldsymbol{\Lambda}}, \hat{\boldsymbol{P}}, \hat{\boldsymbol{Q}}\right) \\
& =\sum_{i=1}^{p^{*}}\left\{\frac{a}{2} \log \frac{a}{2}-\frac{a}{2} \log \left(\frac{1}{\delta_{i}}+\frac{1}{\hat{\lambda}_{i}}\right)-\frac{a}{2}-M \log \hat{\lambda}_{i}\right\} \\
& +\sum_{i=p^{*}+1}^{N}\left\{\frac{a}{2} \log \frac{a}{2}-\frac{a}{2} \log \left(\frac{1}{\delta_{i}}+\frac{1}{\hat{\lambda}_{i}}\right)-\frac{a}{2}\right\} \\
& =\overbrace{\sum_{i=1}^{N}\left(\frac{a}{2} \log \frac{a}{2}-\frac{a}{2}\right)}^{c}-\sum_{i=1}^{p^{*}} \frac{a}{2} \log \left(\frac{1}{\delta_{i}}+\frac{M}{K \delta_{i}}\right) \\
& -M \sum_{i=1}^{p^{*}} \log \left(\delta_{i} \frac{K}{M}\right)-\frac{a}{2} \sum_{i=p^{*}+1}^{N} \log \left(\frac{1+\delta_{i}}{\delta_{i}}\right) \\
& =c-\frac{a}{2} \sum_{i=1}^{p^{*}} \log \left(\frac{K+M}{K \delta_{i}}\right)-M \sum_{i=1}^{p^{*}}\left(\log \delta_{i}+\log \frac{K}{M}\right) \\
& -\frac{a}{2} \sum_{i=p^{*}+1}^{N} \log \left(1+\delta_{i}\right)+\frac{a}{2} \sum_{i=p^{*}+1}^{N} \log \delta_{i} \\
& =c_{1}+\frac{a}{2} \sum_{i=1}^{p^{*}} \log \delta_{i}-M \sum_{i=1}^{p^{*}} \log \delta_{i}+\frac{a}{2} \sum_{i=p^{*}+1}^{N} \log \delta_{i} \\
& -\frac{a}{2} \sum_{i=p^{*}+1}^{N} \log \left(1+\delta_{i}\right) \\
& =c_{1}+K \sum_{i=1}^{p^{*}} \log \delta_{i}+\frac{a}{2} \sum_{i=p^{*}+1}^{N} \log \delta_{i}-\frac{a}{2} \sum_{i=p^{*}+1}^{N} \log \left(1+\delta_{i}\right)
\end{aligned}
$$

where

$$
c_{1}=c-\frac{a}{2} \sum_{i=1}^{p^{*}} \log (K+M)+\frac{a}{2} \sum_{i=1}^{p^{*}} \log K-M \sum_{i=1}^{p^{*}} \log \frac{K}{M} .
$$

Summarizing, the compressed log-likelihood function is given by

$$
\begin{aligned}
-a \log \operatorname{det}(\boldsymbol{B}) & -\frac{a}{2} \sum_{i=1}^{N} \log \delta_{i}+c_{1}+K \sum_{i=1}^{p^{*}} \log \left(\delta_{i}\right) \\
+ & \frac{a}{2} \sum_{i=p^{*}+1}^{N} \log \delta_{i}-\frac{a}{2} \sum_{i=p^{*}+1}^{N} \log \left(1+\delta_{i}\right)
\end{aligned}
$$

whereas the compressed likelihood is

$$
\frac{1}{[\operatorname{det}(\boldsymbol{B})]^{a}} \frac{1}{\prod_{i=1}^{p^{*}} \delta_{i}^{M}} e^{c_{1}} \frac{1}{\prod_{i=p^{*}+1}^{N}\left(1+\delta_{i}\right)^{\frac{a}{2}}} .
$$


As for the optimization problem under $H_{0}$, it is not difficult to show that [23]

$$
\begin{array}{rl}
\max _{\boldsymbol{R}} & f\left(\boldsymbol{Z}_{K}, \boldsymbol{Y}_{M} ; \boldsymbol{R}, H_{0}\right) \\
& =\max _{\boldsymbol{R}}\left[\frac{1}{\operatorname{det}(\boldsymbol{R})}\right]^{K+M} \exp \left\{-\operatorname{tr}\left[\boldsymbol{R}^{-1}(\boldsymbol{G}+\boldsymbol{H})\right]\right\} \\
& =\left[\frac{(K+M)^{N}}{\operatorname{det}(\boldsymbol{G}+\boldsymbol{H})}\right]^{K+M} \exp \{-N(K+M)\} \\
& =\left[(K+M) e^{-1}\right]^{N(K+M)} \frac{1}{[\operatorname{det}(\boldsymbol{G}+\boldsymbol{H})]^{K+M}} \\
& =\left[(K+M) e^{-1}\right]^{N(K+M)}\left[\frac{1}{[\operatorname{det}(\boldsymbol{B})]^{2} \operatorname{det}(\boldsymbol{I}+\boldsymbol{\Delta})}\right]^{K+M} .
\end{array}
$$

Gathering the preceding results, (5) is statistically equivalent to

$$
\Lambda_{p}\left(\boldsymbol{Z}_{K}, \boldsymbol{Y}_{M}\right) \underset{H_{0}}{\stackrel{H_{1}}{\gtrless}} \eta
$$

where

$$
\Lambda_{p}\left(\boldsymbol{Z}_{K}, \boldsymbol{Y}_{M}\right)= \begin{cases}1, & \text { if } p^{*}<p \\ \prod_{i=1}^{p^{*}} \frac{\left(1+\delta_{i}\right)^{K+M}}{\delta_{i}^{M}}, & \text { otherwise. }\end{cases}
$$

\section{APPENDIX II}

\section{DERIVATION OF AN INDUCED MAXIMAL}

$$
\text { INVARIANT FOR } p=1 \text { AND } p=2
$$

Let us focus on the case $p=1$, and then matrix $\boldsymbol{R}_{2}$ can be recast as

$$
\boldsymbol{R}_{2}=|\alpha|^{2} \boldsymbol{p} \boldsymbol{p}^{\dagger}
$$

where $\boldsymbol{p} \in \mathbb{C}^{N \times 1}, \boldsymbol{p} \neq \mathbf{0}$, and $\alpha \in \mathbb{C}, \alpha \neq 0$. As a consequence, the maximum eigenvalue of $\boldsymbol{R}_{1}^{-1}\left(\boldsymbol{R}_{1}+\boldsymbol{R}_{2}\right)$ can be written as

$$
\begin{aligned}
\lambda_{1}\left\{\boldsymbol{R}_{1}^{-1}\left(\boldsymbol{R}_{1}+\boldsymbol{R}_{2}\right)\right\} & =\lambda_{1}\left\{\boldsymbol{I}+|\alpha|^{2} \boldsymbol{R}_{1}^{-1 / 2} \boldsymbol{p} \boldsymbol{p}^{\dagger} \boldsymbol{R}_{1}^{-1 / 2}\right\} \\
& =1+|\alpha|^{2} \lambda_{p}\left\{\boldsymbol{R}_{1}^{-1 / 2} \boldsymbol{p} \boldsymbol{p}^{\dagger} \boldsymbol{R}_{1}^{-1 / 2}\right\} \\
& =1+|\alpha|^{2} \boldsymbol{p}^{\dagger} \boldsymbol{R}_{1}^{-1} \boldsymbol{p}
\end{aligned}
$$

where $\lambda_{p}\{\cdot\}$ is the maximum eigenvalue of $\boldsymbol{R}_{1}^{-1 / 2} \boldsymbol{p} \boldsymbol{p}^{\dagger} \boldsymbol{R}_{1}^{-1 / 2}$. It follows that an induced maximal invariant is

$$
|\alpha|^{2} \boldsymbol{p}^{\dagger} \boldsymbol{R}_{1}^{-1} \boldsymbol{p} .
$$

On the other hand, when $p=2$, the induced maximal invariant is 2-D since, in this case, $\boldsymbol{R}_{2}$ can be written as

$$
\boldsymbol{R}_{2}=|\alpha|^{2} \boldsymbol{p}_{1} \boldsymbol{p}_{1}^{\dagger}+|\beta|^{2} \boldsymbol{p}_{2} \boldsymbol{p}_{2}^{\dagger}=\boldsymbol{P} \boldsymbol{P}^{\dagger} .
$$

Thus, following the preceding line of reasoning, it is possible to show that an induced maximal invariant is a 2 -D vector composed of the eigenvalues of $\boldsymbol{P}^{\dagger} \boldsymbol{R}_{1}^{-1} \boldsymbol{P}$.

\section{ACKNOWLEDGMENT}

The authors would like to thank the National Aeronautics and Space Administration Jet Propulsion Laboratory for providing the SAR data. All data supporting this paper are available from http://uavsar.jpl.nasa.gov.

\section{REFERENCES}

[1] "The dumping of hydrocarbons from ships into the seas and oceans of Europe (The other side of oil slicks)," Oceana, Washington, DC, USA, Tech. Rep., Nov. 2003. [Online]. Available: https://eu.oceana.org/sites/ default/files/reports/oilreport-english.pdf

[2] G. Franceschetti, A. Iodice, D. Riccio, G. Ruello, and R. Siviero, "SAR raw signal simulation of oil slicks in ocean environments," IEEE Trans. Geosci. Remote Sens., vol. 40, no. 9, pp. 1935-1949, Sep. 2002.

[3] P. Lombardo and C. Oliver, "Optimum detection and segmentation of oilslicks using polarimetric SAR data," Proc. Inst. Elect. Eng.-Radar, Sonar Navigat., vol. 147, no. 6, pp. 309-321, Dec. 2000.

[4] W. Wang, F. Lu, P. Wu, and J. Wang, "Oil spill detection from polarimetric SAR image," in Proc. IEEE 10th ICSP, Oct. 2010, pp. 832-835.

[5] R. Shirvany, M. Chabert, and J.-Y. Tourneret, "Ship and oil-spill detection using the degree of polarization in linear and hybrid/compact dual-pol SAR," IEEE J. Sel. Topics Appl. Earth Observ. Remote Sens., vol. 5, no. 3, pp. 885-892, Jun. 2012.

[6] J. Fortuny-Guasch, "Improved oil slick detection and classification with polarimetric SAR," in Proc. POLinsar, ESA-Esrin, Frascat, Italy, Jan. 2003, pp. 1-5.

[7] M. Migliaccio, A. Gambardella, and M. Tranfaglia, "SAR polarimetry to observe oil spills," IEEE Trans. Geosci. Remote Sens., vol. 45, no. 2 , pp. 506-511, Feb. 2007.

[8] P. Lombardo, D. I. Conte, and A. Morelli, "Comparison of optimised processors for the detection and segmentation of oil slicks with polarimetric SAR images," in Proc. IEEE IGARSS, 2000, vol. 7, pp. 2963-2965.

[9] Y. Junjun, Y. Jian, Z. Zheng-Shu, and S. Jianshe, "The extended Bragg scattering model-based method for ship and oil-spill observation using compact polarimetric SAR," IEEE J. Sel. Topics Appl. Earth Observ. Remote Sens., vol. 8, no. 8, pp. 3760-3772, Aug. 2015.

[10] L. Yu, Z. Yuanzhi, C. Jie, and Z. Hongsheng, "Improved compact polarimetric SAR quad-pol reconstruction algorithm for oil spill detection," IEEE Geosci. Remote Sens. Lett., vol. 11, no. 6, pp. 1139-1142, Jun. 2014

[11] A. Solberg, "Remote sensing of ocean oil-spill pollution," Proc. IEEE, vol. 100, no. 10, pp. 2931-2945, Oct. 2012.

[12] S. M. Kay, "The multifamily likelihood ratio test for multiple signal model detection," IEEE Signal Process. Lett., vol. 12, no. 5, pp. 369-371, May 2005.

[13] E. L. Lehmann, Testing Statistical Hypotheses, 2nd ed. New York, NY, USA: Springer-Verlag, 1986.

[14] R. J. Muirhead, Aspects of Multivariate Statistical Theory. New York, NY, USA: Wiley, 1982

[15] S. V. Nghiem, S. H. Yueh, R. Kwok, and F. K. Li, "Symmetry properties in polarimetric remote sensing," Radio Sci., vol. 27, no. 5, pp. 693-711, Sep./Oct. 1992.

[16] J. S. Lee and E. Pottier, Polarimetric Radar Imaging: From Basics to Applications. Boca Raton, FL, USA: CRC Press, 2009.

[17] R. J. Muirhead, Aspects of Multivariate Statistical Theory, vol. 197. New York, NY, USA: Wiley, 2009.

[18] S. M. Kay, "Exponentially embedded families-New approaches to model order estimation," IEEE Trans. Aerosp. Electron. Syst., vol. 41, no. 1, pp. 333-345, Jan. 2005.

[19] V. Carotenuto, A. De Maio, C. Clemente, and J. J. Soraghan, "Invariant rules for multipolarization SAR change detection," IEEE Trans. Geosci. Remote Sens., vol. 53, no. 6, pp. 3294-3311, Jun. 2015.

[20] K. Conradsen, A. A. Nielsen, J. Schou, and H. Skriver, "A test statistic in the complex Wishart distribution and its application to change detection in polarimetric SAR data," IEEE Trans. Geosci. Remote Sens., vol. 41, no. 1, pp. 4-19, Jan. 2003.

[21] L. M. Novak, "Change detection for multi-polarization, multi-pass SAR," in Proc. SPIE Conf. Algorithms Synthetic Aperture Radar Imagery XII, Orlando, FL, USA, Mar. 2005, pp. 234-246.

[22] L. Mirsky, "On the trace of matrix products," Mathematische Nachrichten, vol. 20, pp. 171-174, 1959.

[23] E. J. Kelly, "An adaptive detection algorithm," IEEE Trans. Aerosp. Electron. Syst., vol. 22, no. 2, pp. 115-127, Mar. 1986. 


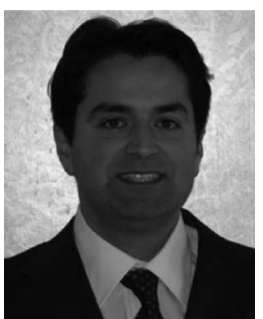

Antonio De Maio (S'01-A'02-M'03-SM'07-F'13) was born in Sorrento, Italy, on June 20, 1974. He received the Dr.Eng.(Hons.) degree and the Ph.D. degree in information engineering from the University of Naples Federico II, Naples, Italy, in 1998 and 2002, respectively.

From October to December 2004, he was a Visiting Researcher with the U.S. Air Force Research Laboratory, Rome, NY, USA. From November to December 2007, he was a Visiting Researcher with the Chinese University of Hong Kong, Hong Kong. He is currently a Professor with the University of Naples Federico II. His research interests lay in the field of statistical signal processing, with emphasis on radar detection, optimization theory applied to radar signal processing, and multiple-access communications.

Dr. De Maio was a recipient of the 2010 IEEE Fred Nathanson Memorial Award as the young (less than 40 years of age) AESS Radar Engineer 2010, whose performance is particularly noteworthy, as evidenced by contributions to the radar art over a period of several years, with the following citation for "robust CFAR detection, knowledge-based radar signal processing, and waveform design and diversity."

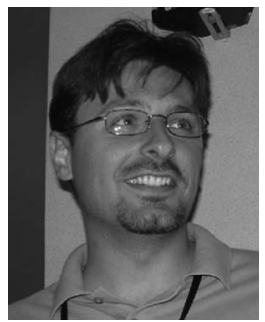

Danilo Orlando (SM'13) was born in Gagliano del Capo, Italy, on August 9, 1978. He received the Dr. Eng.(Hons.) degree in computer engineering and the Ph.D. degree (with maximum score) in information engineering from the University of Salento (formerly University of Lecce), Lecce, Italy, in 2004 and 2008, respectively.

From July 2007 to July 2010, he was with the University of Cassino, Cassino, Italy, where he was engaged in a research project on algorithms for track-before-detect of multiple targets in uncertain scenarios. From September to November 2009, he was a Visiting Scientist with the NATO Undersea Research Center, La Spezia, Italy, where he collaborated with Dr. F. Ehlers on track-before-detect strategies for multistatic sonars. From September 2011 to April 2015, he was with Elettronica SpA, where he was engaged as a System Analyst in the field of electronic warfare. In May 2015, he joined Università degli Studi "Niccolò Cusano," Rome, Italy, where he is currently an Associate Professor. In February-March 2007, he held visiting positions with the Department of Avionics and Systems, École Nationale Supérieure d'Ingénieurs de Constructions Aéronautiques (ENSICA) [now Institut Supérieur de l'Aéronautique et de l'Espace, (ISAE)], Toulouse, France, where he worked with Prof. O. Besson on adaptive radar detection in the presence of mismatched signals. He has authored or coauthored over 70 scientific publications in international journals, conferences, and books. His main research interests are in the field of statistical signal processing and image processing, with greater emphasis on adaptive detection and tracking of multiple targets in multisensor scenarios.

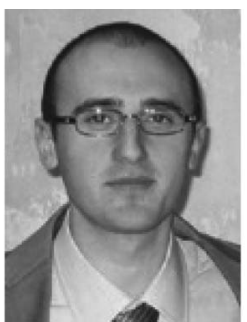

Luca Pallotta (S'12-M'15) received the Laurea Specialistica degree (cum laude) in telecommunication engineering from the University of Sannio, Benevento, Italy, in 2009 and the Ph.D. degree in electronic and telecommunication engineering from the University of Naples Federico II, Naples, Italy, in 2014.

He is currently with the University of Naples Federico II. His research interests lay in the field of statistical signal processing, with emphasis on radar signal processing and radar targets classification.

Dr. Pallotta won the Student Paper Competition at the 2013 IEEE Radar Conference.

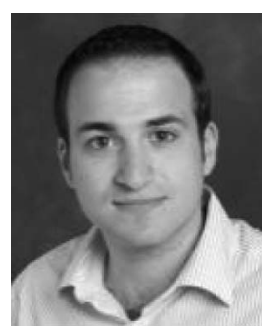

Carmine Clemente (S'09-M'13) received the B.Sc. (cum laude) and the M.Sc. degrees in telecommunications engineering from Universita' degli Studi del Sannio, Benevento, Italy, in 2006 and 2009, respectively, and the Ph.D. degree from the University of Strathclyde, Glasgow, U.K., in 2012.

$\mathrm{He}$ is currently a Lecturer with the Department of Electronic and Electrical Engineering, University of Strathclyde, working on advanced radar signal processing algorithm, multiple-input-multipleoutput radar systems and micro-Doppler analysis. His research interests include synthetic aperture radar (SAR) focusing and bistatic SAR focusing algorithms development, micro-Doppler signature analysis and extraction from multistatic radar platforms, and micro-Doppler classification and statistical signal processing. 